\title{
A novel CARD containing splice-isoform of CIITA regulates nitric oxide synthesis in dendritic cells
}

\author{
Dachuan Huang ${ }^{1}$, Sylvia Lim ${ }^{1}$, Rong Yuan Ray Chua ${ }^{1}$, Hong Shi ${ }^{1}$, Mah Lee $\mathrm{Ng}^{2}$, Siew Heng Wong ${ }^{1 凶}$ \\ ${ }^{1}$ Laboratory of Membrane Trafficking and Immunoregulation, Department of Microbiology, Immunology Programme, Yong Loo \\ Lin School of Medicine, National University of Singapore, Block MD4, 5 Science Drive 2, Singapore 117597, Republic of \\ Singapore \\ ${ }^{2}$ Laboratory of Flavivirology, Department of Microbiology, Immunology Programme, Yong Loo Lin School of Medicine, National \\ University of Singapore, Block MD4, 5 Science Drive 2, Singapore 117597, Republic of Singapore \\ Correspondence: micwsh@nus.edu.sg
}

Received February 16, 2010 Accepted February 25, 2010

\section{ABSTRACT}

MHC class II expression is controlled mainly at transcriptional level by class II transactivator (CIITA), which is a non-DNA binding coactivator and serves as a master control factor for MHC class II genes expression. Here, we describe the function of a novel splice-isoform of CIITA, DC-expressed caspase inhibitory isoform of CIITA (or DC-CASPIC), and we show that the expression of DCCASPIC in DC is upregulated upon lipopolysaccharides (LPS) induction. DC-CASPIC localizes to mitochondria, and protein-protein interaction study demonstrates that DC-CASPIC interacts with caspases and inhibits its activity in DC. Consistently, DC-CASPIC suppresses caspases-induced degradation of nitric oxide synthase2 (NOS2) and subsequently promotes the synthesis of nitric oxide (NO). NO is an essential regulatory molecule that modulates the capability of DC in stimulating $\mathrm{T}$ cell proliferation/activation in vitro; hence, overexpression of DC-CASPIC in DC enhances this stimulation. Collectively, our findings reveal that DC-CASPIC is a key molecule that regulates caspases activity and NO synthesis in DC.

KEYWORDS

dendritic cells, caspase, CIITA, nitric

oxide synthase

\section{INTRODUCTION}

Dendritic cells are a heterogeneous population of cells that play key roles in the immune system, including the establishment of central tolerance in the thymus, the maintenance of self-tolerance in the periphery and the initiation and regulation of adaptive immune responses (Banchereau et al., 2000). The key functions of DC are intimately related to their ability to internalize and process antigens, and to present antigens to T cells. The presentation of peptides to the antigen receptor (TCR) of $\mathrm{CD} 4^{+} \mathrm{T}$ helper lymphocytes is mediated by major histocompatibility complex (MHC) class II molecules.

MHC class II expression is mainly controlled at transcriptional level by a highly conserved regulatory module located at $150-300$ base pairs (bp) upstream of the transcriptioninitiation site (Reith and Mach, 2001; Boss and Jensen, 2003). Enhanceosome binds to this module and serves as a platform for the class II transactivator (CIITA), which is a nonDNA binding coactivator and is a master control factor for MHC class II expression (Reith and Mach, 2001; Ting and Trowsdale, 2002).

The expression of human CIITA is tightly regulated by four different promoters (PI, PII, PIII, PIV) in a cell-dependent manner. The product of PII is absent in mice; PIV is more prominent in thymic epithelial cells (TEC) and nonhematopietic origin cells; PIII is a lymphoid promoter that is active in $\mathrm{B}$ cells and plasmacytoid DCs ( $\mathrm{pDCs}$ ); PI-controlled transcript is specifically expressed in $\mathrm{DC}$ and the translational product is called CIITA type I or DC-CIITA (Muhlethaler-Mottet et al., 1997). In comparison to the products from PIII and PIV, the CIITA protein synthesized from PI has an extra caspase recruitment domain, caspase recruitment domain (CARD)like domain (Nickerson et al., 2001). However, DC-CIITA has a higher transactivation activity than type III CIITA, which does not contain a CARD-like domain. The molecular mechanisms about the function of the CARD-like domain remain largely unknown. The elucidation of the function of the CARD-like domain will be particularly important in the understanding of 
the function of DC because it has been shown that CIITA expression in DC is driven mainly by $\mathrm{PI}$, and at the mRNA level, DC-CIITA transcripts are by far the most abundant mRNA isoform in immature DC (LeibundGut-Landmann et al., 2004).

Here, we describe the function of a novel splice-isoform of DC-CIITA, DC-CASPIC, which mainly encodes for the entire CARD-like domain of DC-CIITA.

\section{RESULTS}

\section{DC-CASPIC is a novel splice-isoform of the DC specific type-I CIITA}

To investigate the underlying mechanisms of the function of the CARD-like domain of DC-CIITA, we first amplified the DNA fragment that encodes for the CARD-like domain of mouse DC-CIITA by reverse-transcriptase-PCR (RT-PCR) using RNA prepared from untreated (control) and LPS-treated DC. The PCR primers were specific to the two flanking ends of the CARD-like domain. Interestingly, we observed two PCR products with distinctive sizes (349 bp and $474 \mathrm{bp}$, respectively) from both the LPS-treated and untreated DC (Fig. 1A). DNA sequencing analysis revealed that both fragments encode for the CARD-like domain of DC-CIITA. However, the larger fragment (DC-CIITA isoform, otherwise known as DC-CASPIC) contains an additional insert of 142 nucleotides. The intensity of the PCR products was quantified, normalized with $\beta$-actin and presented as bar chart (Fig. 1B). Consistent with previous reports (Landmann et al., 2001), we observed a significant reduction in the level of DC-CIITA mRNA upon LPS-induction. Interestingly, the mRNA level of the DCCASPIC did not change in the presence of LPS, indicating that the DC-CIITA-isoform was not regulated at transcriptional
A

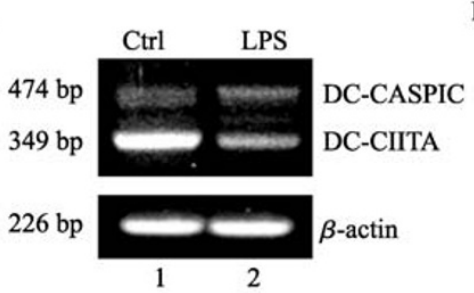

B

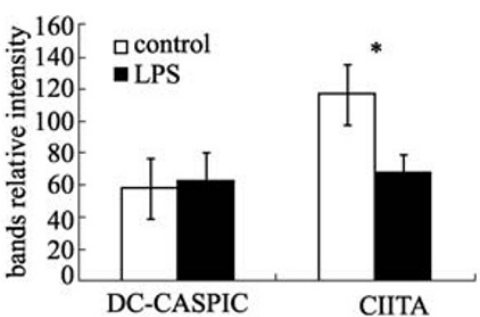

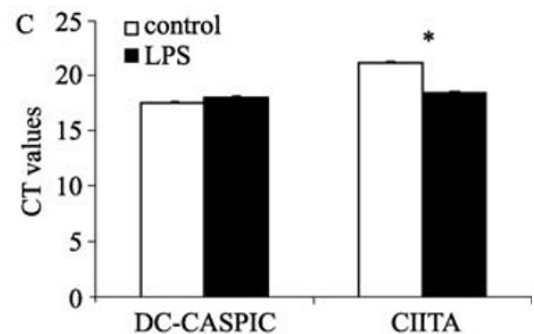

D

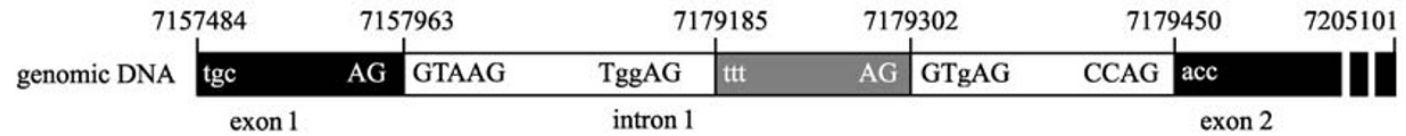

1 transcription
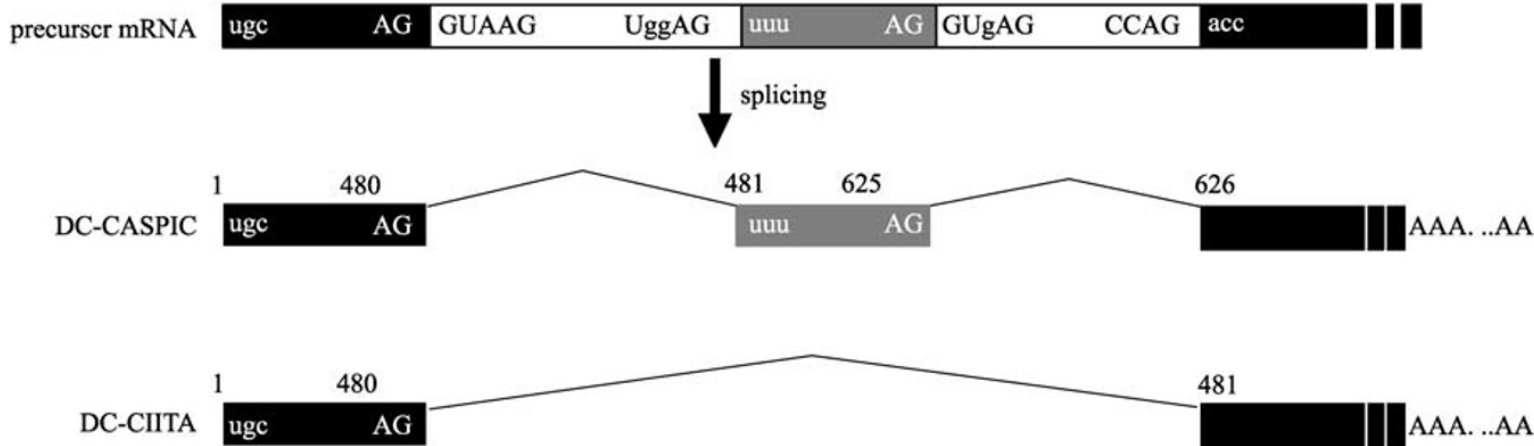

Figure 1. DC-CASPIC is a novel splice-isoform of the DC specific type-I CIITA. (A) Reverse transcription-PCR was performed using untreated (upper panel, lane 1) and LPS-treated DC (upper panel, lane 2) to amplify the cDNA fragment encoding for the CARD-like domain of DC-CIITA. The sizes of the DC-CIITA and CIITA-isoform (DC-CASPIC) are 349 bp and 474 bp, respectively. $\beta$ actin at the lower panel is the loading control. (B) The bars represent the $\beta$-actin-normalized densities of the upper band ( $474 \mathrm{bp}$ ) and lower band (349 bp) shown in panel A. Data shown here are the mean \pm S.D. from three independent cultures. Statistically significant differences (P-value): $*, p<0.05$. (C) Quantitative RT-PCR was performed to confirm the results in panel A. Statistically significant differences (P-value): $*, p<0.05$. (D) In silico mouse genome database search shows that the intron 1 of the mouse DC-CIITA gene contains exon-intron boundaries that agree with consensus splice donor, and thus divides intron 1 (white box) into three parts. Alternative splicing then retains the middle 142 nucleotides intronic sequence (gray box) in the mRNA transcript of the DC-CIITAisoform, which we subsequently named DC-CASPIC. Black boxes represent exons. 
level during DC maturation. These data were further confirmed by quantitative RT-PCR approach. As shown in Fig. $1 \mathrm{C}$, the presence of LPS did not significantly change the mRNA level of DC-CASPIC. As expected, we observed a significant decline of DC-CIITA mRNA expression upon LPSinduction.

In silico mouse genome database search shows that the intron 1 of the mouse DC-CIITA gene contains two pairs of exon-intron boundaries that agreed with the consensus splice donor (Hiller and Platzer, 2008), which divide intron 1 into three parts (Fig. 1D). Alternative splicing then retains the middle 142 nucleotides intronic sequence in the mRNA transcript of DC-CASPIC. As shown in Fig. 2A, the presence of the 142 nucleotides intronic sequence in the DC-CASPIC mRNA transcript created a stop-codon. The mature mRNA of DC-CASPIC and DC-CIITA contains two potential translation initiation codons (AUG-1/AUG-2); whereas the mRNA of CIITA type IV (T4) contains a single potential translational initiation codon (AUG-2). The translation of DC-CIITA and
CIITA type IV (T4) proteins was initiated via AUG-1 and AUG2 , respectively (Muhlethaler-Mottet et al., 1997). Since AUG-1 is upstream of AUG-2, the translation of DC-CASPIC protein is expected to start at AUG-1. Therefore, translation via the AUG-1 is expected to form a polypeptide containing a CARDlike domain (highlighted in yellow) plus an extra stretch of 28 amino acids "VSGAVRDLTSVLGSSLKPSAREASAPNH" (boxed), which has no homology to any of the CIITA forms nor to any known proteins (Fig. 2B). Furthermore, DCCASPIC is rather unique because it does not contain an active common CIITA domain (encoded by exon 2 of the CIITA gene) that is present in all other known CIITA isoforms (DC-CIITA (CIITA type I), CIITA type II, CIITA type III and CIITA type IV (T4)).

To characterize the expression and functions of DCCASPIC, specific antibodies against the unique 28 amino acid sequence at $C$ terminus of DC-CASPIC, were raised in rabbits. To test the specificity of the antibody, Myc-tagged DC-CASPIC plasmid DNA construct (pDC-CASPIC-Myc)
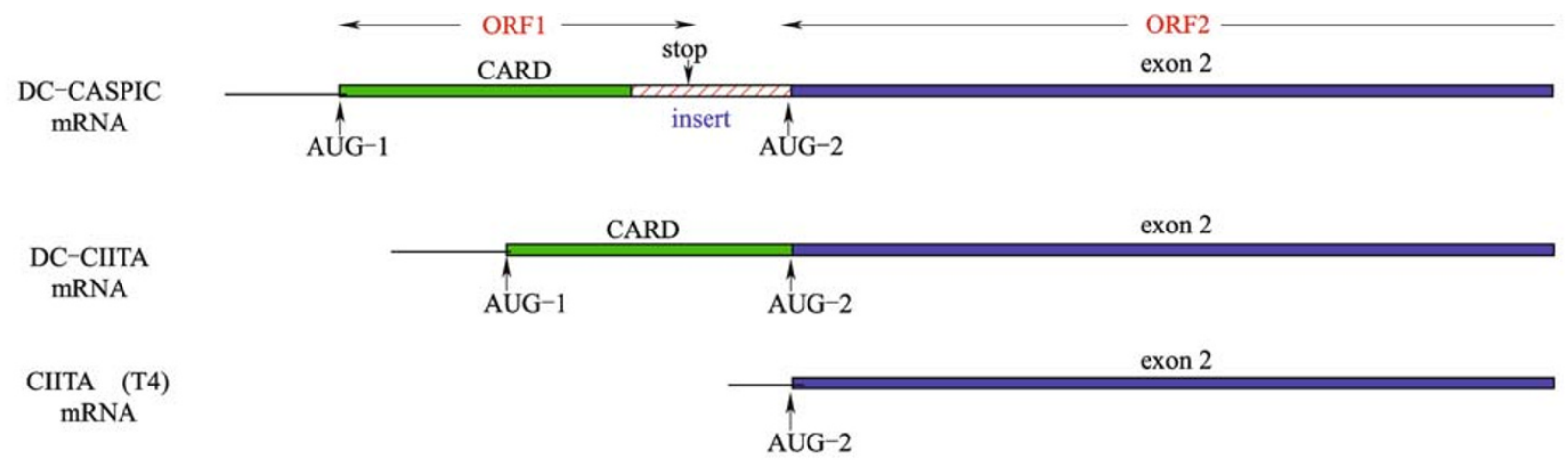

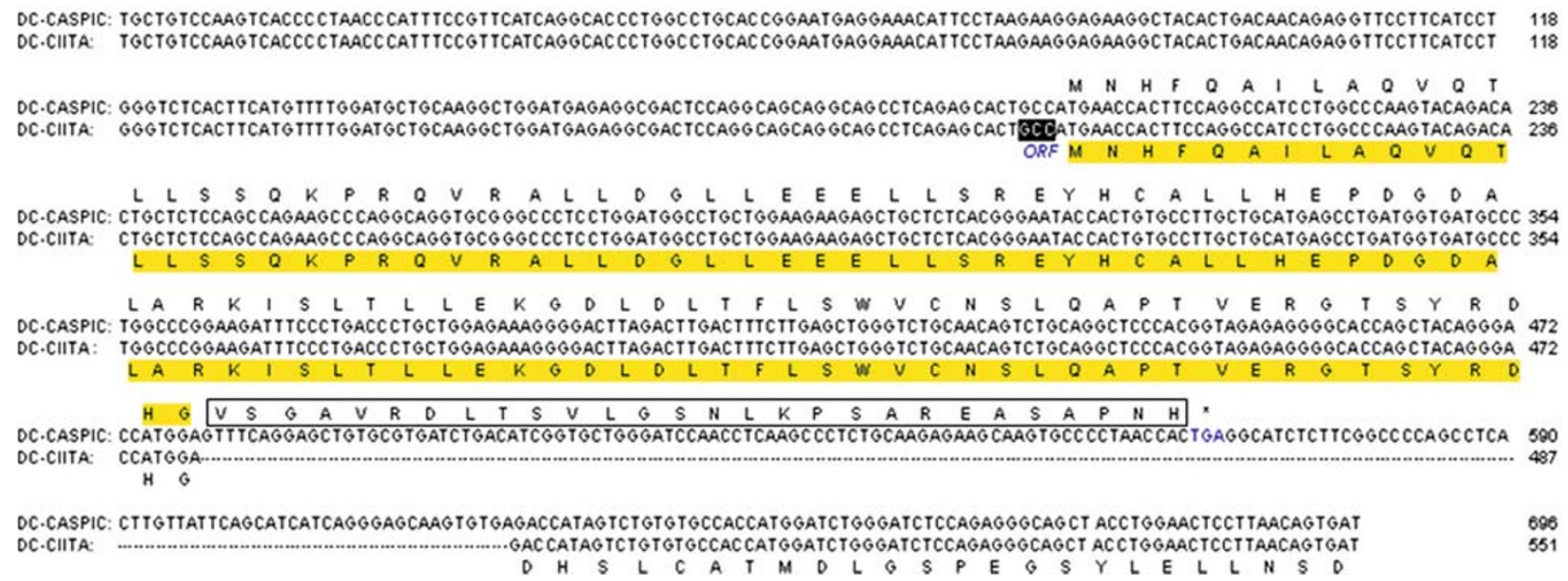

Figure 2. The 142-nucleotide intronic sequence in the DC-CASPIC mRNA transcript creates a stop-codon. (A) Translation via the first AUG formed a polypeptide (ORF1) containing a CARD-like domain (green). DC-CASPIC does not have an active common CIITA domain (encoded by exon 2 of the CIITA gene), which is present in all other known CIITA isoforms (DC-CIITA or CIITA type I, CIITA type II, CIITA type III and CIITA type IV (T4)). (B) In addition to a CARD-like domain (highlighted in yellow), DCCASPIC also contains an extra stretch of C terminus 28 amino acids residues "VSGAVRDLTSVLGSSLKPSAREASAPNH" (boxed), which has no homology to any of the CIITA forms nor to any known proteins. 
was transfected into A-431 cells. Using whole cell extract, the Myc-tagged DC-CASPIC (DC-CASPIC-Myc) proteins were detected by Western blot with the affinity purified DCCASPIC-specific rabbit polyclonal antibody. As shown in Fig. 3A, the antibody detected a protein band (approximately $18 \mathrm{kDa}$ ) in the sample transfected with pDC-CASPIC-Myc plasmid DNA but not in the sample transfected with control pDmyc vector (myc). Moreover, the detection of the DCCASPIC-Myc protein band could be blocked by the immunogen, a synthetic peptide composed of the unique 28 amino acids found at the C-terminus of DC-CASPIC, thus confirming the specificity of the antibody. We next determined the expression of endogenous DC-CASPIC proteins in mouse-, rat- and human-derived cell lines by Western blot using the DC-CASPIC specific polyclonal antibody. As shown in Fig. 3B, the expression of DC-CASPIC was detected in the mouse $\mathrm{DC}(\mathrm{DC} 2.4$ and primary $\mathrm{DC}(\mathrm{pDC}))$, mouse microglia cells (N9), mouse macrophages (RAW), mouse myoblasts (C2C12), human epithelial cells (Hela, A-431 and Caco-2), human monocytes (THP-1) and human T-lymphocytes (Jurkat). Because DC, macrophages and microglial cells expressed DC-CASPIC, it is interesting to determine whether DC-CASPIC is induced by bacterial LPS. To examine this possibility, mouse bone marrow-derived primary DC were either untreated or treated with LPS, and the protein expression of DC-CASPIC was determined. As shown in Fig. 3C, DC-CASPIC was upregulated upon LPS induction, suggesting the potential significance of DC-CASPIC in DC and other antigen presenting cells. Consistently, immunohistochemical staining of mouse brain sections showed that DCCASPIC is preferentially expressed in MHC II-positive cells (presumably the microglia cells) of the brain (Fig. 4A and 4B). DC-CASPIC also has higher expression in CD11c-positive cells of the lamina propria of colon (Fig. 4C-E) and small
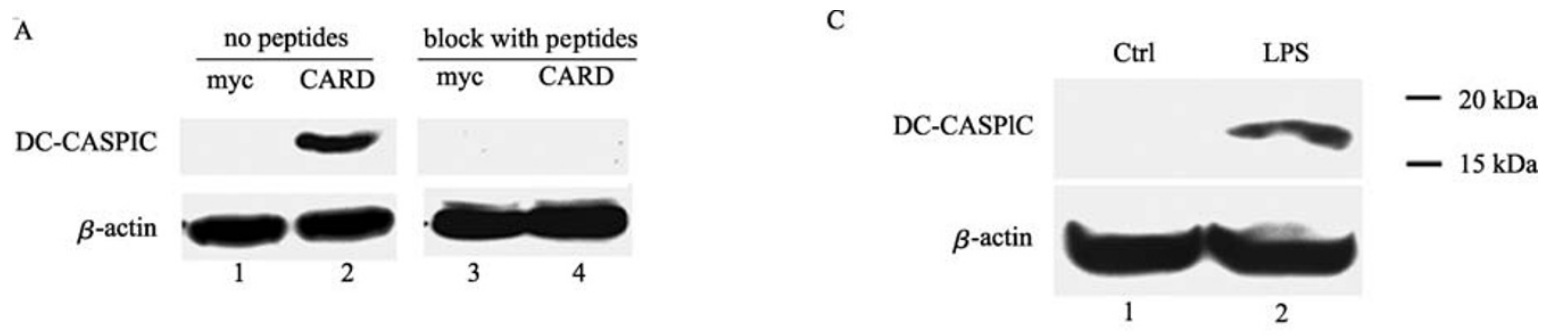

B
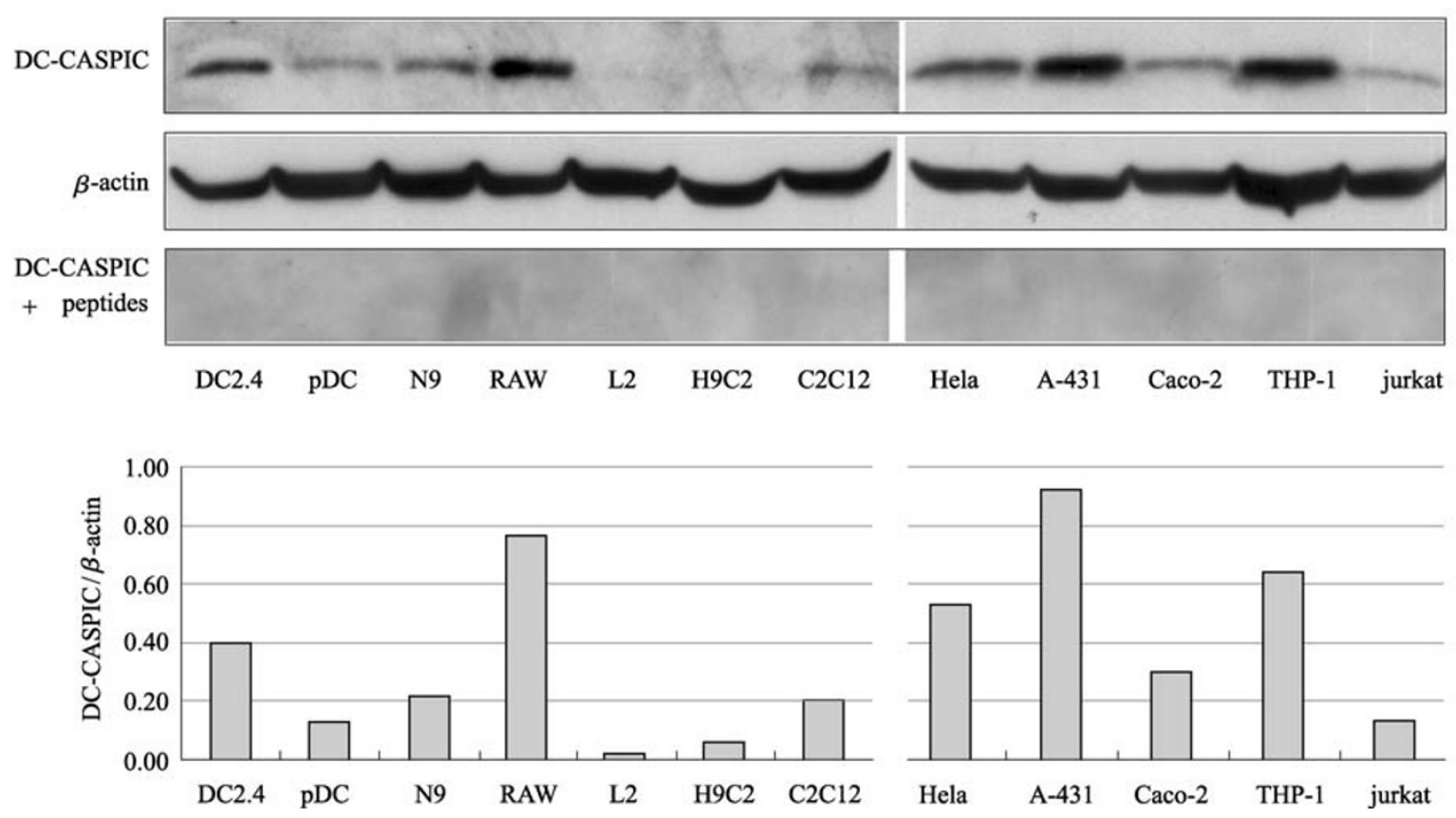

Figure 3. DC, microglial and macrophages express DC-CASPIC. (A) Cell extracts from A-431 cells that were transfected with either empty pDMyc vector or pDC-CASPIC-Myc were probed with the DC-CASPIC (in the presence or absence of blocking immunogenic peptide) and $\beta$-actin antibodies. (B) Eighty micrograms $(80 \mu \mathrm{g})$ of protein extracts derived from indicated cells were separated on SDS-PAGE and analyzed by Western blotting using anti-DC-CASPIC- and anti- $\beta$-actin antibodies in the presence or absence of blocking immunogenic peptide. (C) DC 2.4 cells were treated with $1 \mu \mathrm{g} / \mathrm{mL}$ LPS for $48 \mathrm{~h}$ or untreated, and the cell extracts were analyzed by Western blot using anti-DC-CASPIC and anti- $\beta$-actin antibodies. 
intestine (Fig. 4F-H). These DC-CASPIC ${ }^{+} \mathrm{CD}_{11 \mathrm{C}^{+}}$cells are most likely $D C$ that infiltrated into the lamina propria from the surrounding blood vessels (Arques et al., 2009).

\section{DC-CASPIC localizes to mitochondria}

To further characterize DC-CASPIC, we next determined the subcellular localization of DC-CASPIC. We chose A-431 cells over DC in this subcellular localization analysis due to its larger size and higher transfection efficiency. Plasmid cDNA construct pDC- CASPIC-Myc was transfected into A-431 cells, and the expression of DC-CASPIC-Myc fusion protein was detected with anti-Myc antibody and analyzed by confocal immunofluorescence microscopy. As shown in Fig. 5A, DC-CASPIC-Myc proteins were detected on cytoplasmic punctate membraneous structures (panel a), and interestingly, these structures colocalized well with mitochondrial cytochrome $c$ proteins (panel b and c). Importantly, similar membraneous structures were also observed in the MHC II-positive (microglial) cells of the brain (Fig. 4B). These data indicate that DC-CASPIC localizes to cytoplasm and mitochondrial organelle. The localization of DC-CASPIC in mitochondria was further verified by immunogold electron microscopy. DC2.4 cells were fixed and stained with antibodies against DC-CASPIC and cytochrome $c$ followed by $10 \mathrm{~nm}$ and $5 \mathrm{~nm}$ immunogold-conjugated secondary antibodies, respectively. As shown in Fig. 5B, DC-CASPIC proteins $(10 \mathrm{~nm}$ immunogold, green arrowhead) were detected in both mitochondrial organelle as marked by cytochrome $c(5 \mathrm{~nm}$ immunogold, red arrow) and the adjacent cytoplasm (star). To further verify the subcellular localization of DC-CASPIC, mitochondria were isolated from DC by cell fractionation. Protein extracts from either total cell (total) or mitochondria (Mit) were separated by SDS-PAGE followed by Western blot analysis with specific antibodies against DC-CASPIC, cytochrome c, c-Myc and TFIIB. Consistently with previous observations, DC-CASPIC was enriched in mitochondrial fraction (Fig. 5C, lane 8). The C-Myc and TFIIB protein served as cytoplasm and nucleus markers, respectively.
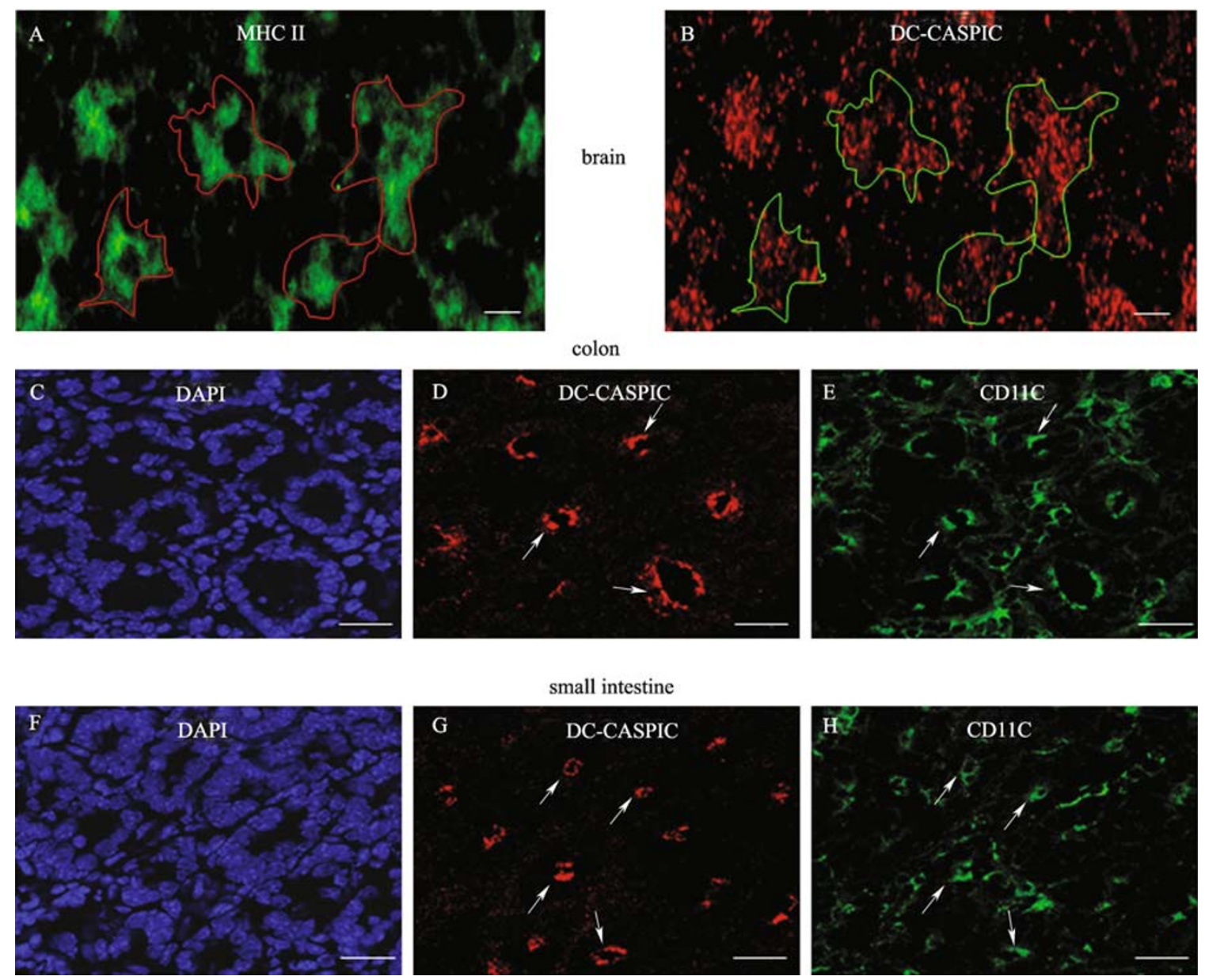

Figure 4. DC-CASPIC protein is detected in the brain, colon and small intestine. Mouse brain (A and $B)$, colon (C, D, and E) and small intestine $(F, G$, and $H)$ tissue sections were labeled with anti-DC-CASPIC and either anti-MHC II or anti-CD11C antibodies, and analyzed by confocal microscopy. Scale bar: $10 \mu \mathrm{m}(\mathrm{A}$ and $\mathrm{B}), 30 \mu \mathrm{m}(\mathrm{C}-\mathrm{H})$. 

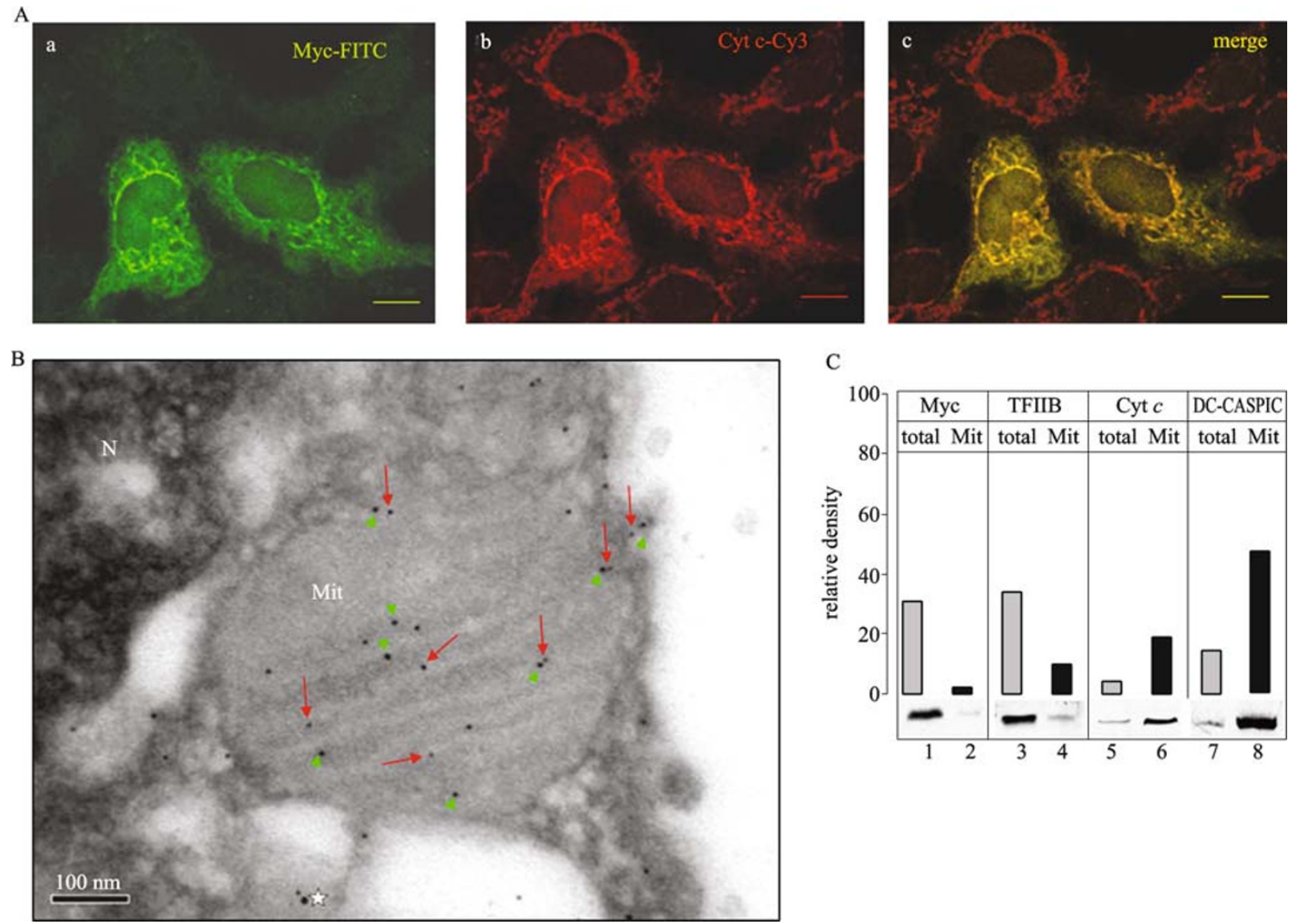

C

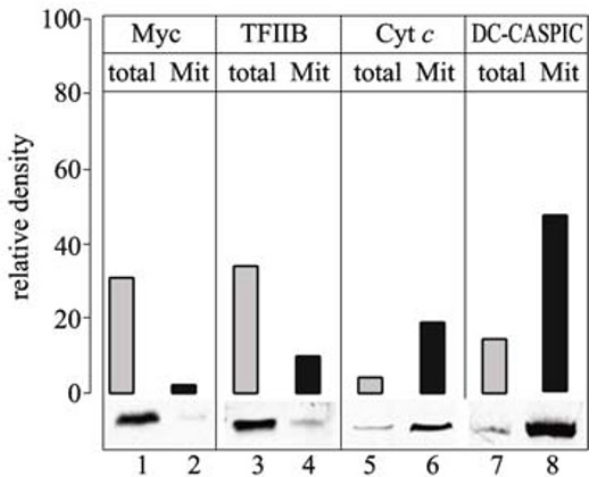

Figure 5. DC-CASPIC localizes to mitochondria. (A) A-431 cells transfected with DC-CASPIC-Myc were fixed and stained with specific antibodies against c-Myc and cytochrome $c$ (Cyt $c$ ), followed by FITC- and cy3-conjugated secondary antibodies, respectively. DC-CASPIC-Myc and cytochrome $c$ were subsequently detected under a confocal immunofluorescence microscopy (Scale bar: $5 \mu \mathrm{m}$ ). (B) Ultrathin sections of DC2.4 cells were fixed and stained with antibodies against cytochrome $c$ and DC-CASPIC followed by $5 \mathrm{~nm}$ and $10 \mathrm{~nm}$ immunogold-conjugated secondary antibodies, respectively. DC-CASPIC (green arrowhead) and cytochrome $c$ (red arrow) was subsequently detected by electron microscopy (Mit: mitochondria, N: nuclear, Star: cytoplasm, Scale bar: $100 \mathrm{~nm}$ ). (C) Protein extracts from primary DC whole cells (total) or mitochondria (Mit) was separated by SDS-PAGE and probed with Myc, TFIIB, cytochrome $c$ and DC-CASPIC antibodies. Bars represent the $\beta$-actin-normalized density of the correspondence protein bands. Data shown are representative of at least two independent experiments with similar results.

\section{a-helices $\mathrm{H} 3$ and $\mathrm{H} 4$ on DC-CASPIC are required for mitochondrial localization}

The N-terminus of DC-CASPIC is identical to the previously reported CARD-like domain of DC-CIITA (Nickerson et al., 2001). To examine whether an intact CARD-like domain is required for its mitochondrial localization, plasmid DNA constructs encoding for three truncated myc-tagged DCCASPIC proteins (DC-CASPIC(1-34)-Myc, DC-CASPIC (1-69)-Myc and DC-CASPIC(1-95)-Myc), which include amino acid residue 1-34, 1-69 and 1-95, respectively, were generated (Fig. 6A). These plasmid DNA constructs were individually transfected into A-431 and DC2.4 cells. The transfected cells were fixed, stained with anti-c-Myc and anticytochrome $c$ antibodies, and analyzed by confocal immunofluorescence microscopy. As shown in Fig. 6B and 6C (panel a and b), DC-CASPIC(1-95)-Myc and DC-CASPIC (1-69)-Myc colocalized well with mitochondria cytochrome $c$ proteins. However, little or no colocalization with cytochrome $c$ was observed for DC-CASPIC(1-34)-Myc (Fig. 6B and $6 \mathrm{C}$, panel C). Colocalization of DC-CASPIC(1-95)-Myc protein with cytochrome $c$ was further confirmed by $3 D \mathrm{X}-\mathrm{Z} / \mathrm{Y}$ $\mathrm{Z}$ confocal image projections (Fig. 6B, panel d). Therefore, deletion of $\mathrm{H} 3$ and $\mathrm{H} 4$ in DC-CASPIC(1-34)-Myc abrogates mitochondrial localization. Localization of DC-CASPIC(1-69)Myc (which lacks $\mathrm{H} 5$ and $\mathrm{H} 6$ ) to mitochondria suggests that an intact (whole) CARD-like domain is not essential for mitochondrial localization and amino acid residue 1-69 of DC-CASPIC is sufficient for targeting of DC-CASPIC to mitochondria. 
A

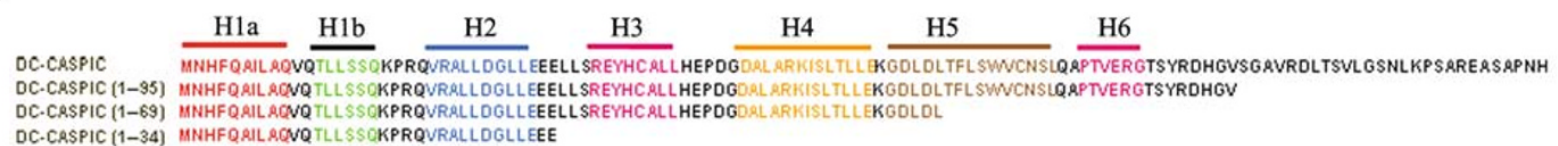
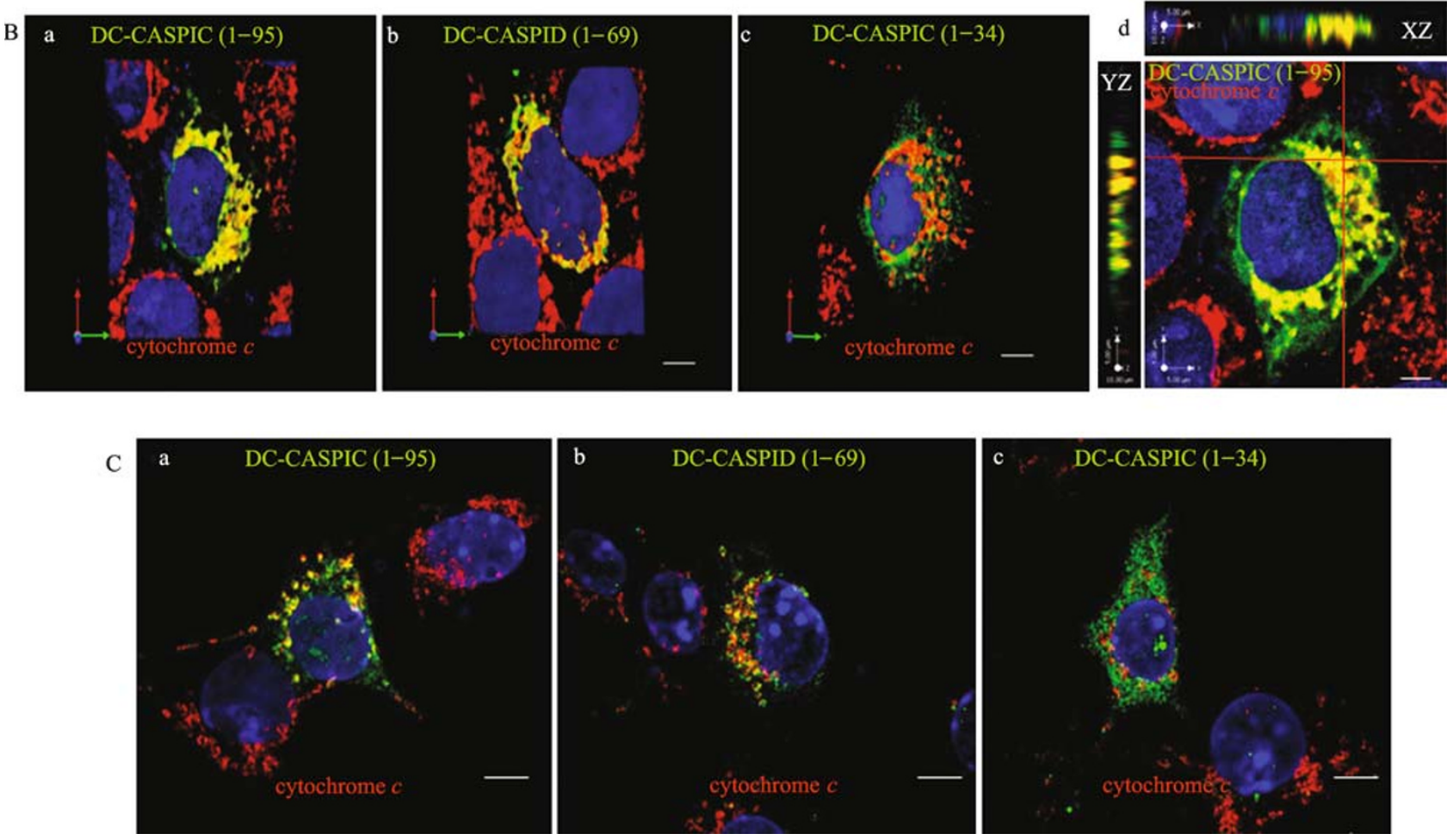

Figure 6. $\alpha$-helices $\mathrm{H} 3$ and $\mathrm{H} 4$ on DC-CASPIC are required for mitochondrial localization. (A) Generation of DC-CAPSIC truncated constructs. H1a-H6 represents the six typical a-helices of the CARD domain (Nickerson et al., 2001). The truncated proteins are labeled as DC-CASPIC (1-34), DC-CASPIC (1-69) and DC-CASPIC (1-95). (B) Various DC-CASPIC truncated constructs were transfected into A-431 cells. The cells were fixed and doubly labeled with c-Myc and cytochrome $c$ antibodies, followed by FITC and cy3-conjugated secondary antibodies. The nucleus was stained by DAPI (blue). DC-CASPIC and cytochrome $c$ were subsequently analyzed by confocal immunofluorescence microscopy (Scale bar: $5 \mu \mathrm{m}$ ). Co-localization of DC-CASPIC and cytochrome $c$ was shown in yellow. Panel $d$ is the $X Z / Y Z$ projection of the confocal images (Improvision Volocity software) showing colocalization of DC-CASPIC(1-95)-Myc and cytochrome $c$ in mitochondria. Co-localization of DC-CASPIC and cytochrome c was shown in yellow. Scale bar: $5 \mu \mathrm{m}$. (C) DC-CASPIC truncated constructs were transfected into DC2.4 cells. The cells were fixed and doubly labeled with c-Myc and cytochrome $c$ antibodies, followed by FITC and cy3-conjugated secondary antibodies. The nucleus was stained by DAPI (blue). DC-CASPIC and cytochrome $c$ were subsequently detected by confocal immunofluorescence microscopy. Colocalization of DC-CASPIC and cytochrome $c$ was shown in yellow. Scale bar: $5 \mu \mathrm{m}$.

\section{DC-CASPIC induces NOS2 expression and NO synthesis in DC}

The upregulation of DC-CASPIC during DC maturation suggests its potential functional significance in DC. To determine the roles of DC-CASPIC in DC, DC2.4 cells were transfected with vector control (pDMyc) and pDC-CASPIC$\mathrm{Myc}$, and treated with LPS. The release of NO (one of the key molecules produced by DC during maturation) from the untreated and LPS-treated transfected cells was measured. Fig. 7A shows that DC-CASPIC significantly enhanced synthesis of NO in mature (LPS-treated) DC. To determine whether the whole CARD-like domain of DC-CASPIC is required for the induction of NO synthesis, same amounts of vector control (pDMyc), pDC-CASPIC-Myc, pDC-CASPIC (1-95)-Myc, pDC-CASPIC(1-69)-Myc or pDC-CASPIC (1-34)-Myc plasmid DNA constructs were transfected into DC2.4 cells and the NO production was examined. As shown in Fig. 7B (panel a), except for DC-CASPIC(1-34)-Myc, all other DC-CASPIC constructs significantly induced NO production in the LPS-treated DC2.4 cells. Fig. 7B (panel b) showed the $\beta$-actin-normalized Myc-tagged protein expression level in transfected DC2.4 cells. DC-CASPIC(1-34)-Myc, which lacks $\alpha$-helices $\mathrm{H} 3$ and $\mathrm{H} 4$, lost its ability to enhance $\mathrm{NO}$ synthesis. These data indicate that amino acid residue 35-68 ( $\alpha$-helices $\mathrm{H} 3$ and $\mathrm{H} 4$ ) of DC-CASPIC is required for inducing NO. In accompany with this $\mathrm{NO}$ induction, we also observed an increase in NOS2 protein expression in 
A

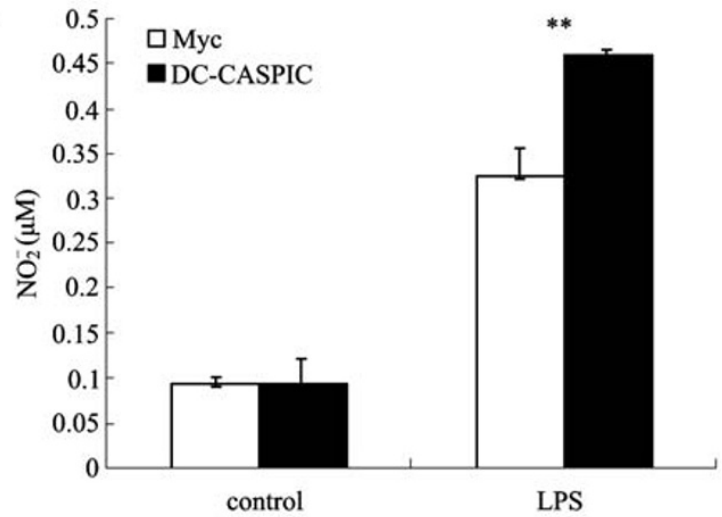

C

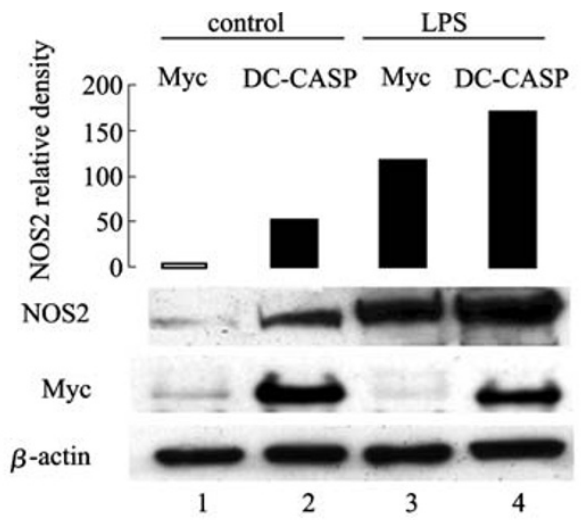

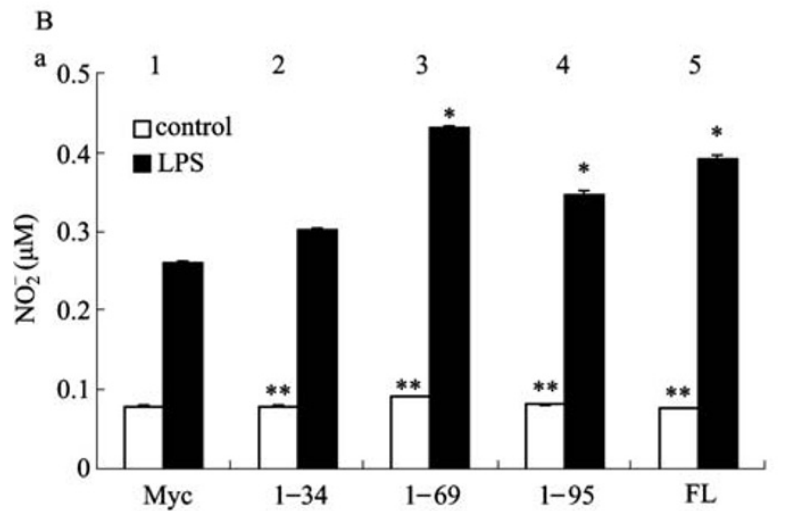

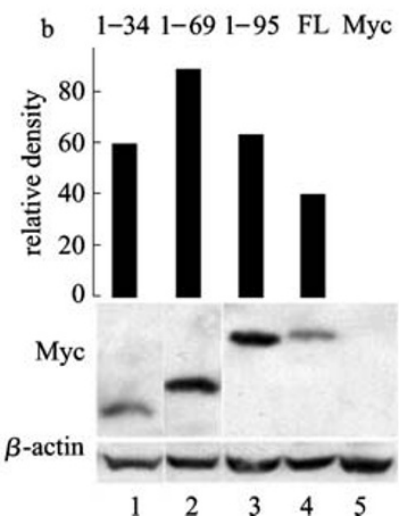

D

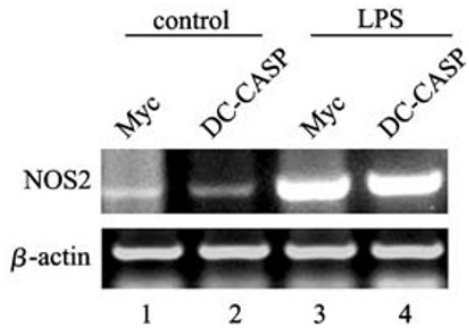

Figure 7. DC-CASPIC induces NOS2 expression and NO synthesis in DC. (A) DC2.4 cells were stably transfected with pDMyc empty vector (white bars) or pDC-CASPIC-Myc (black bars), and either treated with $1 \mu \mathrm{g} / \mathrm{mL}$ LPS for $48 \mathrm{~h}$ or untreated. The NO production was measured by a Griess assay. Data shown are the mean \pm S.D. of triplicate samples and are representative of at least two independent experiments with similar results. Statistically significant differences (P-value): $* *, p<0.01$. (B) DC2.4 cells were transfected with either pDMyc empty vector, pDC-CASPIC(1-34)-Myc, pDC-CASPIC(1-69)-Myc, pDC-CASPIC(1-95)-Myc or pDCCASPIC-Myc plasmid, and were either treated (black bar) with $1 \mu \mathrm{g} / \mathrm{mL}$ LPS for $48 \mathrm{~h}$ or untreated (white bar). (a) The NO production was measured by a Griess assay. Data shown here are the mean \pm S.D. from three independent experiments. Statistically significant differences (P-value) between mutants (lane 2-5) and c-Myc (lane 1): $*, p<0.05$. (b) Cell lysates were subjected to a Western blot analysis using anti-Myc monoclonal antibodies. $\beta$-actin was used as loading control. (C) The pDMyc vector and pDC-CASPIC-Myc plasmids were transfected into DC2.4 cells and treated with LPS or untreated. Cells were lysed and subjected to Western blot analysis with Myc, NOS2 and $\beta$-actin antibodies. The bars represent the $\beta$-actin-normalized densities of NOS 2 bands in the Western blot. Data shown are representative of at least two independent experiments with similar results. (D) mRNA was isolated from the same cells as prepared in panel C, and the mRNA expression of NOS2 and $\beta$-actin was determined by RT-PCR.

DC-CASPIC-transfected DC2.4 cells (Fig. 7C); while this upregulation of NOS2 protein was not due to an increase in NOS2 mRNA transcripts, as shown by RT-PCR in Fig. 7D.

\section{DC-CASPIC inhibits caspase activity}

Since the DC-CASPIC-induced upregulation of NOS2 protein expression was not regulated at mRNA level, we hypothesized that NOS2 might be a substrate for proteases in cytoplasm. In silico analysis of the amino acid sequence of NOS2 revealed a number of conserved potential caspase recognition/cleavage sites (unpublished data). To determine whether NOS2 is a substrate for caspases, immature primary DC were either untreated or treated with synthetic caspaseinhibitor $(\mathrm{Cl})$, and the expression of NOS2 was measured. As shown in Fig. 8A, Cl-treatment upregulated the protein level of NOS2, thus indicating that NOS2 is a substrate for caspases. NO-donor (NONOate), which inhibits caspases-induced protein degradation, was utilized as positive control (Kim et al., 2002; Brüne, 2003; Huang et al., 2008).

Because DC-CASPIC contains a CARD-like domain and DC-CASPIC induces NOS2 expression, we hypothesized that DC-CASPIC can directly inhibit caspases activity in DC. To prove this hypothesis, cell extracts from primary DC, which 


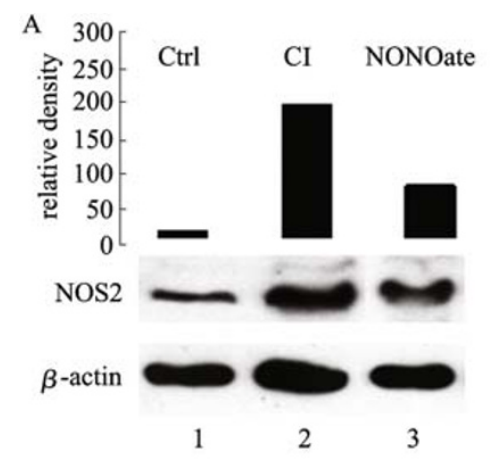

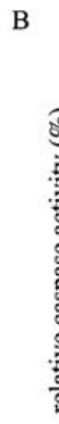

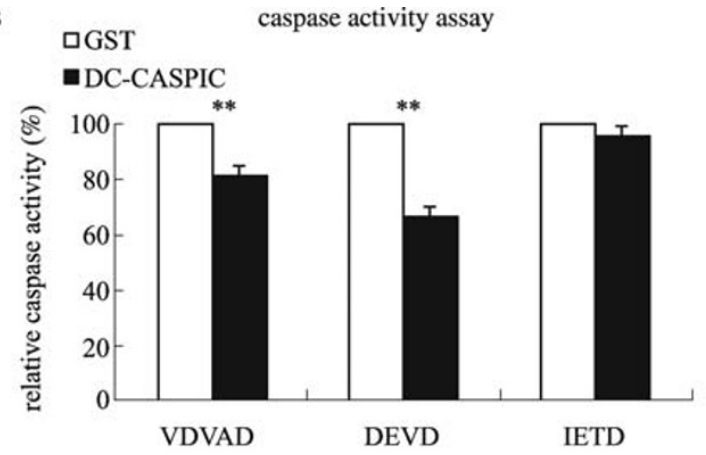

$\mathrm{C}$

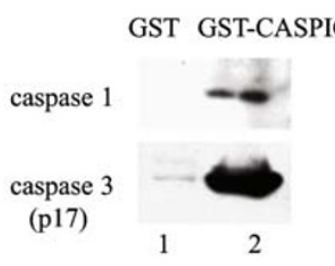

$\mathrm{D}$
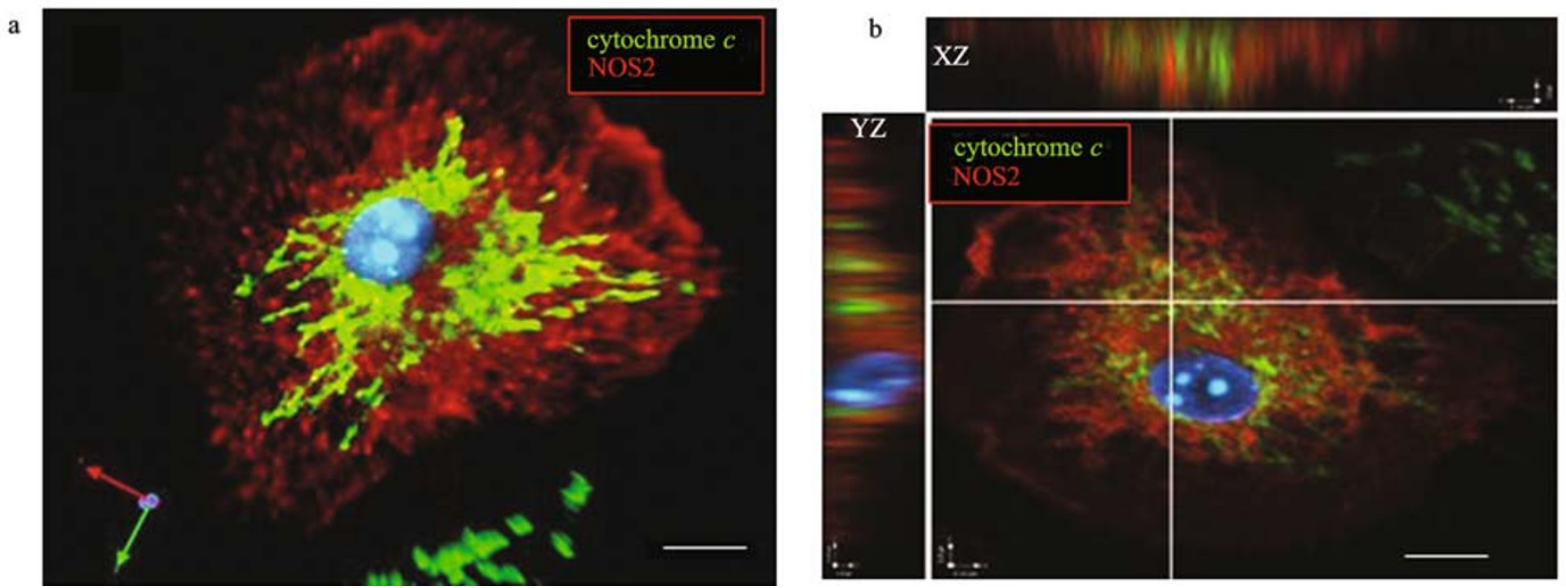

Figure 8. NOS2 is a substrate for caspases and DC-CASPIC inhibits caspase activity. (A) DC2.4 cells were untreated (lane 1), treated with $200 \mathrm{nM}$ caspase inhibitor (Cl) for $4 \mathrm{~h}$ (lane 2), or treated with $200 \mu \mathrm{M}$ NONOate for $4 \mathrm{~h}$ (lane 3). The cells were then lysed and subjected to Western blot analysis with NOS2 and $\beta$-actin antibodies. (B) Cell extract $(300 \mu g)$ from primary DC was incubated for $4 \mathrm{~h}$ with $1 \mu \mathrm{g}$ of GST or GST-DC-CASPIC protein, together with caspase 1 substrate ( $p$-YVAD), caspase 3 substrate ( $p$ DEVD) or caspase 8 substrate ( $p$-IETD), respectively. The caspase activities were measured as described in Materials and Methods. The caspase activity in control, which was incubated with GST protein, was set as $100 \%$. Statistically significant differences (P-value): $* *, p<0.01$. (C) The CARD domain at the $\mathrm{N}$ terminus of DC-CASPIC was cloned into a pGEX-KG vector and expressed as GST-tagged CARD domain (GST-DC-CASPIC). GST (lane 1) or GST-DC-CASPIC (lane 2) was used as bait to pull down potential interacting partners from the total DC cell extract, followed by Western blot analysis using caspase- 1 , and caspase-3 antibodies. (D) Primary DC were fixed with methanol and stained with antibodies against cytochrome $c$ and NOS2, followed by FITC and cy3-conjugated secondary antibodies, respectively. The nucleus was stained by DAPI. NOS2 and cytochrome $c$ were subsequently detected by confocal immunofluorescence microscopy. (a) Stereo 3D rendering images (Improvision Volocity software) of cytochrome $c$ (green) and NOS2 (red) subcellular localization in DC. (b) XZ/YZ projection (Improvision Volocity software) of collected confocal images showing subcellular localization of NOS2 and cytochrome $c$ in DC. Scale bar: $10 \mu \mathrm{m}$.

has high caspase activity (Wong et al., 2004), were incubated for $4 \mathrm{~h}$ with either GSTor a GST-DC-CASPIC protein, together with caspase- 1 substrate ( $p$-YVAD), caspase- 3 substrate ( $p$ DEVD) or caspase-8 substrate ( $p$-IETD), respectively. Then caspase activity was analyzed using a fluorescence reader. As shown in Fig. 8B, GST-DC-CASPIC (CARD-like domain (amino acid 1-95) of DC-CASPIC fused to GST), but not GST, significantly reduced the activity of caspase- 1 and caspase-3 in the primary DC extract. Since DC-CASPIC inhibits caspase activity in DC, it is possible that DC-CASPIC interacts with caspases through its CARD-like domain. To determine this possibility, a protein pull-down assay using GST-DC-CASPIC as bait was performed. Interestingly, the CARD-like domain of DC-CASPIC forms a complex with caspase- 1 and caspase-3 (Fig. 8C). Collectively, our results show that DC-CASPIC inhibits caspases, protects NOS2 from degradation and subsequently induces NO synthesis.

Next, we explored whether NOS2 also localized in mitochondria. However, 3D rendering and $\mathrm{XZ} / \mathrm{YZ}$ projection of confocal images showed that NOS2 colocalized only partially with cytochrome $c$ and the major pool of the NOS2 in DC was found in cytoplasm (Fig. 8D). 


\section{DC-CASPIC enhances DC-dependent T cell proliferation/ activation in vitro}

We have previously shown that NO is the key molecule that regulates the expression of cell surface $\mathrm{MHC} \mathrm{II}$ and the antigen presentation in DC (Huang et al., 2008). Since DCCASPIC is upregulated during DC maturation and it can induce NOS2 expression and NO synthesis, we hypothesized that DC-CASPIC also has important roles in DC functions. To investigate the roles of DC-CASPIC, primary immature DC were transfected with in vitro transcribed mRNA (by electroporation), and stained by MHCII-, CD80- and CD86-specific antibodies prior to flow-cytometry analysis. As shown in Fig. 9A, DC-CASPIC modestly upregulates the surface expression of MHC II, CD80 and CD86, which are markers
DC maturation. To determine whether DC-CASPIC could enhance DC-dependent T cell proliferation in vitro, DC2.4 cells were transfected with either pDMyc control vector or pDC-CASPIC-Myc, chemically fixed and incubated with $T$ cells in a mixed lymphocyte reaction (MLR). At day 3 , IL-2 was produced by Tcells and secreted into the culture medium, and were quantified by ELISA. As shown in Fig. 9B (panel a), DCCASPIC enhanced DC-dependent T cell proliferation (IL-2 level) in the absence and presence of LPS, indicating that DC-CASPIC (like LPS and NO-donor) enhances the capability of DC in inducing T cell proliferation/activation. Fig. 9B, panel $b$ confirmed the expression of DC-CASPIC-Myc proteins in DC2.4 transfected cells. Taken together, our results strongly suggest that DC-CASPIC is one of the key molecules that regulate $\mathrm{NO}$ synthesis during DC maturation

A
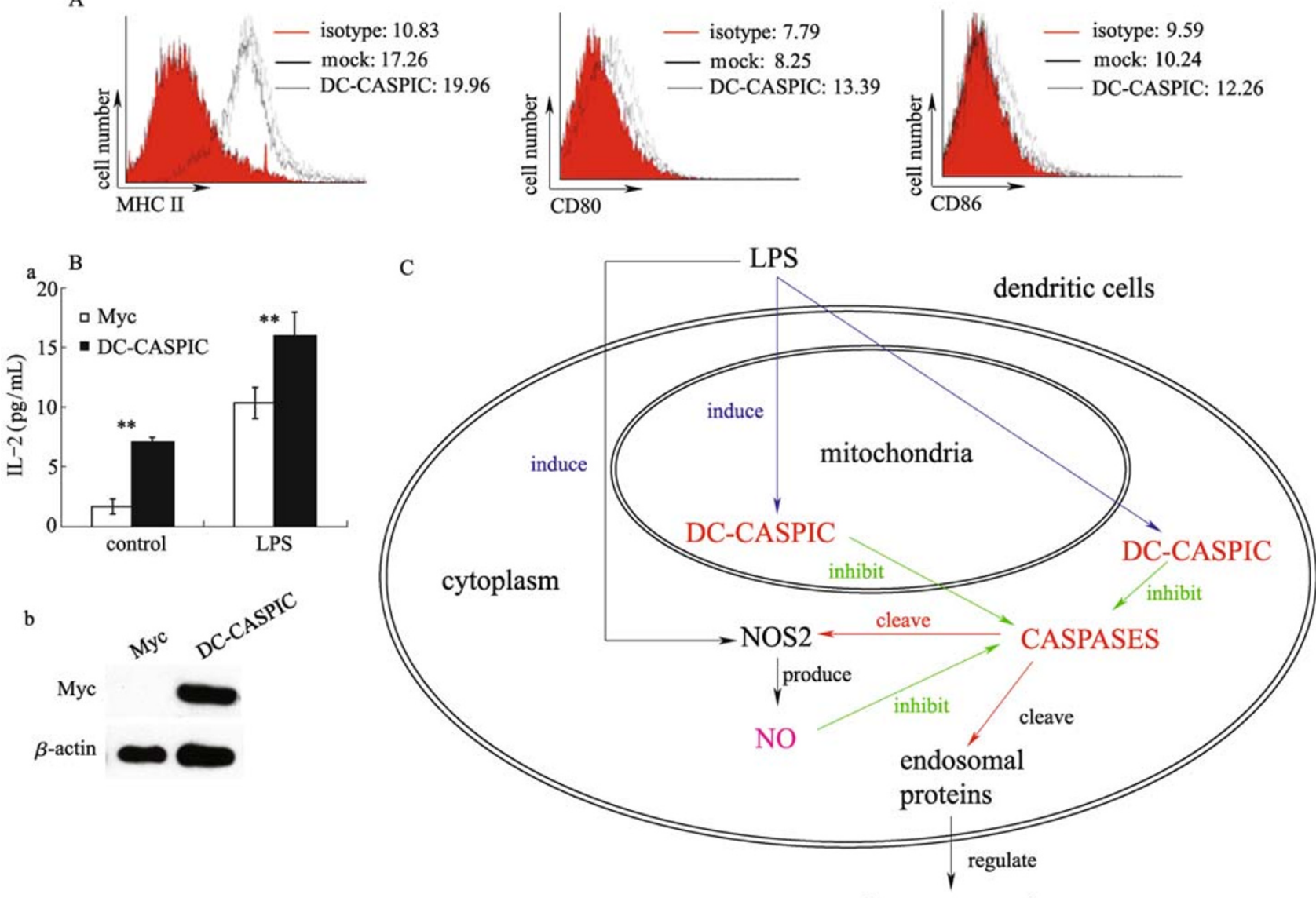

antigen presentation

Figure 9. DC-CASPIC enhances DC-dependent T cell activation/proliferation. (A) Immature DC were transfected with in vitro transcribed mRNA encoding DC-CASPIC. DC were stained with MHCII-FITC, CD80-FITC or CD86-FITC antibodies, and analyzed by flow-cytometry. Control staining (red histograms) was performed with the corresponding isotype lgG. (B) DC2.4 cells were either transfected with a pDMyc empty vector or with DC-CASPIC-Myc. These cells were induced with $1 \mu \mathrm{gg} / \mathrm{mL}$ LPS for $24 \mathrm{~h}$ or untreated, and subsequently cocultured with T cells isolated from BALB/c mice for $72 \mathrm{~h}$. (a) The IL-2 level in media was measured. (b) Cell lysates were subjected to Western blot analysis with Myc and $\beta$-actin antibodies. Statistically significant differences ( $P$-value): $* *, p<0.01$. (C) Cross-talk among DC-CASPIC, caspases, NOS2 and NO regulates the ability of DC in inducing T cell proliferation and activation. 
and the ability of DC in inducing $\mathrm{T}$ cell proliferation/ activation.

\section{DISCUSSION}

In our model (Fig. 9C), the presence of bacterial LPS upregulates protein expression of DC-CASPIC, which then interacts with caspases (presumably in cytoplasm because we did not detect caspases in mitochondria (unpublished data)), and interestingly, inhibits caspases activity. The inhibition of caspases indirectly upregulates protein expression of NOS2, a caspase substrate (also presumably in cytoplasm because of the low expression level of NOS2 in mitochondria), and thus, increases intracellular NO synthesis. NO then further inhibits caspases and enhances NOS2 expression and NO synthesis in mature DC. We have shown previously that inhibition of caspases by $\mathrm{NO}$ upregulates expression of CD74 (Huang et al., 2008) and other endosomal proteins (Wong et al., 2004; Santambrogio et al., 2005), which is essential for regulating the MHC IIrestricted antigen presentation pathway. This subsequently enhances the capability of DC in inducing T cell proliferation/ activation. Collectively, our study shows that DC-CASPIC plays important roles in regulating caspases activity, NO synthesis and antigen presentation in DC.

DC-CASPIC is a splice-isoform of DC-CIITA that is transcribed via the type-I CIITA promoter. The presence of 142 nucleotides intronic sequence created a stop-codon and thus formed a truncated DC-CIITA that only retains the CARD-like domain. Since CIITA synthesized via promoters II, III, and IV is functional even in the absence of the CARD-like domain, DC-CASPIC containing only the CARD-like domain is expected to have distinct functions. The DC-CASPIC mRNA contains two major open reading frames (ORFs) (Fig. 2A). One encodes for the entire CARD (ORF1, translated via AUG-1) and the other (ORF2 or exon 2, translated via AUG-2) encodes for the common CIITA domain. The start codons of both ORFs were preceded by conserved kozak's translation start sites (Kozak, 2001, 2002), and thus, ORF1 was a functional upstream ORF (uORF). Although it is uncommon in eukaryotes, UORF may play a role in repressing translation of low abundance proteins, such as the angiotensin II and the corticotrophin releasing hormone type-I receptor (Mori et al., 1996; Xu et al., 2001). uORF is also reported to be essential in regulating the translation of $\mathrm{c}$ myc proto-oncogene, which encodes for a nuclear phosphoprotein that regulates crucial genes for cell proliferation and differentiation (Newman et al., 1996; Newman and William, 1997). Therefore, it is expected that the translation of exon 2 or ORF2 (that encodes for the functional CIITA protein) from the DC-CASPIC mRNA transcript is also repressed by ORF1. Since DC-CASPIC is a splice-isoform of DC-CIITA and both DC-CASPIC and DC-CIITA mRNA shared the same precursor-mRNA transcribed from the CIITA type I promoter, changes to the DC-CASPIC/DC-CIITA mRNA ratio is expected to alter the overall activity of CIITA in DC. This could be a mechanism for DC to regulate the expression of $\mathrm{MHC}$ genes.

DC-CASPIC proteins were also detected in non-DC cells, such as macrophages (monocytes), microglial cells, T lymphocytes and epithelial cells. Unlike DC-CIITA, which is DC-specific, DC-CASPIC is ubiquitously expressed. Therefore, it is possible that DC-CASPIC have broad physiological significance in various cell types and tissues. Since both DCCASPIC and DC-CIITA were derived from the same precursor mRNA, it is possible that the regulation of splicing in DC is different from other cell types. Hence, it is likely that DC expressed both DC-CASPIC and DC-CIITA mRNA isoforms; whereas other cell types only expressed the DC-CASPIC mRNA isoform. Further study using RT-PCR to detect and to quantify these two mRNA isoforms in non-DC cell types will shine more lights on this issue.

CIITA has been well-characterized as a non-DNA binding transcription factor. This study reveals for the first time that a splicing isoform of the DC-CIITA plays important roles in the regulation of NOS2 expression and NO synthesis. In spite of the low homology to CARD domain, we show that DCCASPIC interacts with caspase-1 and caspase-3. Since caspase-3 does not contain a CARD domain and DCCASPIC inhibits caspase activity, it is possible that DCCASPIC binds to the catalytic region of caspases and inhibits its activity. Alternatively, it is also possible that DC-CASPIC can interact (via its CARD-like domain) with other CARDcontaining proteins that are capable of binding to the catalytic region of caspase-3. Our results also demonstrate that DCCASPIC localizes to both mitochondria and cytoplasm. However, caspases are mainly found in cytoplasm. Thus, it is possible that the mitochondria pool of DC-CASPIC may have other unknown functions. Further study is required to elucidate these important issues.

Mitochondria are the essential organelles for cell bioenergetics. During the past years, it became evident that NO has significant regulatory roles on mitochondrial respiration due to its high-affinity binding to cytochrome oxidase (COX), the final electron acceptor (Brown, 1995; Boveris et al., 1999). There are three major isoforms of NOS, NOS1 (neuronal NOS or nNOS), NOS2 (inducible NOS or iNOS) and NOS3 (or the endothelial NOS/eNOS) (Nathan, 1992). In addition, new isoforms or mitochondrial variants of NOS (mtNOS) were recently described in rat liver (Ghafourifar and Richter, 1997; Giulivi et al., 1998), thymus (Bustamante et al., 2000) and brain (Riobo et al., 2002). It was previously reported that mtNOS forms complexes with mitochondrial COX (Persichini et al., 2005; Franco et al., 2006) and complex I (Franco et al., 2006), thus favoring a steric relationship of newly synthesized $\mathrm{NO}$ to the control of mitochondrial-dependent respiration reaction. Interestingly, the mtNOS had been reported to be a NOS1 isoform that is translocated to mitochondria. Like 
NOS1, mtNOS is constitutively expressed, and its activity is $\mathrm{Ca}^{2+}$-dependent and inducible by drugs/hormones (Carreras et al., 2001; Riobo et al., 2002; Boveris et al., 2003; Carreras et al., 2004). Unexpectedly, a large pool of NOS2 was localized in cytoplasm and little in mitochondria. Therefore, the DC-CASPIC-dependent upregulation of cytoplasmic NOS2 could be due to the inhibition of cytoplasmic caspases by cytoplasmic pool of DC-CASPIC.

Generally, newly synthesized DC-CASPIC proteins are localized to cytoplasm. How is DC-CASPIC then targeted to mitochondria while DC-CASPIC does not have any conserved or typical mitochondria-targeting signal? One possibility is that DC-CASPIC interacts with a protein that contains a mitochondria-targeting motif in cytoplasm. Binding of DCCASPIC to this protein could be possibly via its CARD-like domain. Since CARD-CARD interaction is a common phenomenon (Hong and Jung, 2002), it is possible that the interacting partner of DC-CASPIC contains a CARD-like motif as well. However, localization of DC-CASPIC(1-69)-Myc (which lacks $\mathrm{H} 5$ and $\mathrm{H} 6$ ) to mitochondria suggests that an intact (whole) CARD-like domain is not essential for mitochondrial localization. It is possible that CARD-like domain is required for optimal mitochondrial recruitment although amino acid residue 1-69 of DC-CASPIC is sufficient for targeting DC-CASPIC to mitochondria.

Interestingly, despite that DC-CASPIC could induce NOS2 protein expression in immature DC (Fig. 7C, lane 1 and 2), we could not detect the increase of NO in the medium of DCCASPIC-transfected DC in the absence of LPS (Fig. 7A). Since the DC-CASPIC-induced NOS2 protein level in immature DC is relatively low, it is likely that the synthesized NO was still intracellularly localized and we could not detect the change of NO concentration in the medium of DCCASPIC-transfected immature DC.

In spite of the extensive work on CIITA to date, the subcellular localization of DC-CIITA that possesses CARDlike domain has not been reported before.We observed that DC-CASPIC localizes to mitochondria, and thus, DC-CIITA is expected to localize to mitochondria as well. Interestingly, our study shows that like other CIITA, DC-CIITA localizes to cytoplasm and nucleus but not mitochondria (unpublished data). It has been reported that the C-terminal two-third of the CIITA protein contains at least two nuclear-localization signals (NLS2 and NLS3) (Cressman et al., 2001). Thus, it is possible that these signals in the full length DC-CIITA might cover mitochondria targeting signal in the CARD-like domain. In addition, it is also possible that mitochondrial targeting signal in the CARD-like domain could be covered by the secondary and tertiary protein folding in the full-length DCCIITA molecule.

Other than induction of MHC genes by DC-CIITA, NO synthesis is also (Huang et al., 2008) essential for DC maturation. Consistently, our study shows that a novel DCCIITA splicing isoform, DC-CASPIC, regulates the expression of NOS2 and NO synthesis in the maturing DC. This is an efficient mechanism where transcription via the type-1 CIITA promoter produces two splice-isoforms of mRNA encoding for two proteins (DC-CIITA and DC-CASPIC) with distinct functions in the maturing DC, where DC-CIITA preferentially localizes to nucleus (Cressman et al., 2001) and regulates transcription of MHC genes while DC-CASPIC localizes to mitochondrial organelle (as well as cytoplasm) and regulates NO synthesis.

\section{MATERIALS AND METHODS}

\section{Mice}

C57BL/ 6J $\left(I-A^{d}, I^{d} E^{d}\right)$ were from center for animal resources Singapore. All protocols on mice are conducted under the approval of the International Animal Care and Use Committee (IACUC), National University of Singapore.

\section{Cell Culture}

DC2.4 cell line was kindly provided by Dr. Kenneth Rock. DC2.4 cells display dendritic morphology, express dendritic cell-specific markers, MHC molecules and co-stimulatory molecules, and have phagocytic activity and antigen-presenting capacity. A-431 human epidermoid carcinoma cells (CRL-1555), Raw 264.7 mouse monocytes/macrophages cell line (TIB-71), L2 rat lung epithelial cell line (CCL-149), H9C2 rat myoblastic cell line (CRL-1446), C2C12 mouse myoblastic cell line (CRL-1771), Hela human adenocarcinoma epithelial cell line (CCL-2), Caco-2 human epidermoid carcinoma cell line (HTB-37), THP-1 human monocytes/macrophage cell line (TIB-202), and Jurkat T cell lymphocyte cell line (TIB-152) were obtained from the American Type Culture Collection (ATCC, Manassas, VA, USA). N9, a wellcharacterized murine microglial cell line was a kind gift from Dr. Ricciardi-Castagnoli. Bone marrow-derived DC from 6- to 7-week-old C57BL/6J mice was established by the bulk-culture method as previously described (Wong et al., 2004). Briefly, bone-marrow cells were harvested from the long bones and plated on dishes in complete DMEM with $10 \%$ FCS containing $20 \mathrm{ng} / \mathrm{mL}$ of mouse recombinant GM-CSF (R\&D Systems, USA). Cells were fed every $2 \mathrm{~d}$ with complete DMEM containing $15 \mathrm{ng} / \mathrm{mL}$ GM-CSF. All of cells were maintained in DMEM supplemented with $5 \%$ FCS in a $37^{\circ} \mathrm{C}$ incubator with $5 \% \mathrm{CO}_{2}$.

\section{Plasmid constructs}

The cDNA for cloning of DC-CASPIC was generated using MLV-RT reverse transcriptase (Promega) with oligo dT as primers. Total RNA from BMDC was isolated using the TRIzol reagent (Invitrogen). The cDNA was further amplified by PCR using forward primer (5'GGAGAATTCAATGAACCACTTCCAGGCCATCCTG-3') and reverse primer (5'-GCTCTAGAGGTCAGTGGTTAGGGGCACTTGCTTC TCTTG-3'). The DNA fragment generated was then subcloned into pDMyc mammalian cell expression vector by insertion into the EcoRI and $X b a l$ cloning site. For the other 3 deletion mutants, the same cloning strategy was performed, except that different reverse primers were used: 5'-GCTCTAGAGGTCACTCTTCTTCCAGCAGGCC-3' 
for DC-CASPIC (1-34); 5'-GCTCTAGAGGTCACAAGTCTAAGTCCCCTTTCTC-3' for DC-CASPIC (1-69) and 5' -GCTCTAGAGGTCAAACTCCATGGTCCCTGTAGC-3 for DC-CASPIC (1-95).

The GST-CARD was generated by PCR with forward primer (5'GCGCGGATCCGCGAACCACTTCCAGGCCATCCTG-3') and reverse primer (5' -GCCGGAATTCCGTCAGTCCCTGTAGCTGGTG$\left.3^{\prime}\right)$. The resulting DNA fragment was cloned into $\mathrm{PGEX-KG}$ vector (Pharmacia) at BamH1 and EcoR1 restriction sites to establish the cDNA plasmid construct pGEX-KG-GST-CARD.

\section{Protein expression and purification}

Briefly, pGEX-KG-GST-CARD construct was transformed into BL21 (DE3) E. coli competent cells. Bacteria was incubated at $37^{\circ} \mathrm{C}$ in LB and induced with $0.5 \mathrm{mM} I P T G$ for $4 \mathrm{~h}$. Cell pellets were resuspended and sonicated in extraction buffer $(150 \mathrm{mM} \mathrm{NaCl}, 20 \mathrm{mM}$ Tris- $\mathrm{HCl}, \mathrm{pH}$ 7.4, $2 \mathrm{mM}$ EDTA, $2 \mathrm{mM}$ DTT, $1 \%$ Triton X-100 and a cocktail of protease inhibitors). The supernatant was then subjected to glutathione-sepharose purification as described by the manufacturer (Amersham).

\section{Antibodies}

DC-CASPIC specific rabbit polyclonal antibody was raised against the unique $C$ terminus of DC-CASPIC by Pacific Immunology Corp (CA). Synthetic peptide "VSGAVRDLTSVLGSNLKPSAREASAPNH" was used as immunogen.

\section{Protein pull-down}

DC2.4 cells were lysed at $4^{\circ} \mathrm{C}$ with extraction buffer $(150 \mathrm{mM} \mathrm{NaCl}$, $20 \mathrm{mM}$ Tris-HCl, $\mathrm{pH} 7.4,2 \mathrm{mM}$ EDTA, $2 \mathrm{mM}$ DTT and a cocktail of protease inhibitors) containing $1 \%$ Triton $\mathrm{X}-100$. The extracts were incubated with GST fusion proteins immobilized on the beads overnight at $4^{\circ} \mathrm{C}$. Beads were then washed twice with extraction buffer containing $0.5 \%$ Triton $\mathrm{X}-100$, twice with extraction buffer containing $0.1 \%$ Triton $\mathrm{X}-100$, and once with extraction buffer without Triton $X-100$. Proteins were separated on SDS-PAGE gel and analyzed by Western blot using indicated antibodies.

\section{Co-immunoprecipitation}

A-431 cells were transfected with indicated constructs, and $1 \mathrm{mg}$ of protein extracts were incubated with polyclonal anti-myc antibodies or rabbit IgG $(10 \mu \mathrm{g})$ bound protein A Sepharose beads (Pharmacia) overnight in IP buffer (150 mM NaCl, $20 \mathrm{mM}$ Tris-HCl, pH 7.4, $2 \mathrm{mM}$ EDTA, $2 \mathrm{mM}$ DTT, $1 \%$ Triton $\mathrm{X}-100$ and protease inhibitors) at $4^{\circ} \mathrm{C}$. Beads were then washed twice in buffer $A$ (identical to IP buffer except that it contains $0.5 \%$ Triton $\mathrm{X}-100$ ) and twice in buffer $\mathrm{B}$ (identical to IP buffer except that it contains $0.1 \%$ Triton X-100) before re-suspended in SDS sample buffer. Immunoprecipitated proteins were separated on SDS-PAGE and analyzed by immunoblot using indicated antibodies.

\section{Immunofluorescence}

Cells grown on coverglasses were fixed in pre-chilled methanol for $4 \mathrm{~min}$ at $-20^{\circ} \mathrm{C}$ then transferred to room temperature for $2 \mathrm{~min}$. The coverglasses were then washed (with rocking for 2-3 min) four times with PBS, and then incubated with primary antibodies followed by secondary antibodies (fluorescein- or Cy3-conjugated) for about $1 \mathrm{~h}$ each at room temperature. Non-specific binding was removed by washing (with rocking for 2-3 min) three times with PBS before mounting and viewing under the microscope. The conventional fluorescent images were taken using Olympus BX-60 digital microscope with ImagePro Plus software. The confocal images were taken using Olympus Fluoview 500 microscope with Fluoview version 5.0 software or Zeiss LSM 510 Meta (upright stage) with Zeiss LSM Image Browser version 4.0.0.091 software.

\section{Reverse-transcriptase PCR (RT-PCR) and quantitative RT-PCR}

Total RNA was extracted from untreated and LPS-treated primary DC and CDNA were synthesized by reverse-transcription using oligo-dT primers. PCR were performed using DC-CASPIC and DC-CIITA specific primers. PCR products of DC-CASPIC (474 bp) and DCCIITA (349 bp) were analyzed on an agarose gel and quantified. Quantitative RT-PCR was performed using the ABI SYBR green PCR mix and ABI7500 system. GADPH forward and reverse primers were used as control.

\section{Immunohistochemistry}

For immunofluorescence analysis on frozen sections, C57BL/6J mouse organs were dissected, embedded in OCT, frozen and cryosectioned $(6-8 \mu \mathrm{m})$ using the Leica Cryostat. Sections were fixed in $4 \%$ paraformaldehyde for $30 \mathrm{~min}$, washed with $0.1 \%$ TritonPBS (PBST) 10 min for three times, blocked with 4\% BSA in PBS for $1 \mathrm{~h}$ and then washed with PBST $10 \mathrm{~min}$ three times. Sections were incubated with indicated primary antibodies for $1 \mathrm{~h}$ at room temperature, followed by washing with PBST $10 \mathrm{~min}$ three times. Sections were then incubated with secondary antibodies for $1 \mathrm{~h}$ at room temperature, followed by washing with PBST for $10 \mathrm{~min}$ three times. Sections were mounted with mounting medium with DAPI. Images were captured with Leica TCS SP5 laser-scanning confocal microscopy or Olympus Fluoview 1000 laser-scanning confocal microscopy.

\section{Western blotting analysis}

Proteins resolved by SDS-PAGE were electroblotted to a nitrocellulose membrane (Hybond) and incubated overnight at $4{ }^{\circ} \mathrm{C}$ with blocking buffer (PBS containing 5\% skim milk and 0.05\% Tween-20). Primary and secondary antibody incubations were performed in blocking buffer. The membrane was washed with PBS containing $0.05 \%$ Tween-20, and then analyzed using the Supersignal Chemiluminescent Kit (Pierce) according to the manufacturer's recommendation.

\section{Flow-cytometry analysis}

Cells were harvested with PBS supplemented with 2 mM EDTA, then washed once with staining buffer (5\% ( $w / v)$ BSA, 2 mM EDTA, 2 mM $\mathrm{NaN}_{3}$ in PBS) followed by incubation for 30 min on ice with the FITCconjugated antibody. For intracellular staining, cells were fixed and permeabilised with pre-chilled methanol for $2 \mathrm{~min}$, then incubated with primary antibody followed by PE conjugated anti-rabbit IgG. After 
washing with staining buffer, cells were fixed with $1 \%(w / v)$ paraformaldehyde and the immunophenotypic analysis was performed on a FACSCAN flow cytometry (DAKO).

\section{In vitro transcription of capped mRNA and transfection of} primary DC by electroporation

Plasmid cDNA constructs were linearized by digestion with an appropriate restriction endonuclease followed by purification with QIAquick gel extraction kit (Qiagen). The in vitro transcription was performed with T7 polymerase according to the manufacturer's instruction (Promega). After performing the in vitro transcription reaction, the mRNA was purified through phenol extraction followed by ethanol precipitation. The RNA concentration was determined by absorbance at $260 \mathrm{~nm}$ and visualized by denaturing gel electrophoresis.

For mRNA transfection, primary DCs were harvested at day 6 with $2 \mathrm{mM}$ EDTA, washed twice with serum-free Opti-MEM, and resuspended to a final concentration of $4 \times 10^{7}$ cells $/ \mathrm{mL}$ in Opti-MEM medium. Subsequently, $100 \mu \mathrm{L}$ cell suspension was mixed with $20 \mu \mathrm{g}$ mRNA and electroporated in a $0.2 \mathrm{~cm}$-cuvette (voltage: $300 \mathrm{~V}$, capacitance: $150 \mu \mathrm{F}$, resistance: $100 \Omega$ ) using the GenePulser II apparatus (Bio-Rad). After electroporation, the cells were transferred to fresh culture medium containing GM-CSF or induced with $1 \mu \mathrm{g} / \mathrm{mL}$ LPS. The cells were cultured for $24 \mathrm{~h}$ to allow full maturation.

\section{Caspase activity assay}

Ten million cells were lysed with $1 \mathrm{~mL}$ lysis buffer (50 mM HEPES, $5 \mathrm{mM}$ DTT, $0.1 \mathrm{mM}$ EDTA, $0.1 \%(v / v)$ CHAPS, $\mathrm{pH} 7.4)$ for $4 \mathrm{~min}$ at $4^{\circ} \mathrm{C}$. Twenty microgram of protein was incubated at $37^{\circ} \mathrm{C}$ in a buffer containing $25 \mathrm{mM}$ HEPES ( $\mathrm{pH} 7.5), 10 \%(w / v)$ sucrose, $0.1 \%(\mathrm{v} / \mathrm{v})$ CHAPS and $10 \mathrm{mM}$ DTT, with the respective colorimetric substrates (caspase-1 substrate ( $p$-YVAD), caspase-3 ( $p$-DEVD), and caspase8 substrate ( $p$-IETD)) (all from Calbiochem), in a 96-well flat-bottom microtiterplate. The cleavage of the substrates was quantified after $2 \mathrm{~h}$ by measuring the absorbance at $405 \mathrm{~nm}$.

\section{Measurement of NO}

NO synthesis was evaluated by measuring the concentration of stable end product $\mathrm{NO}_{2}{ }^{-}$, which was determined by Griess reaction. Aliquots of culture supernatant $(100 \mu \mathrm{L})$ were incubated with $100 \mu \mathrm{L}$ of Griess reagent (Sigma) at room temperature for $10 \mathrm{~min}$. The absorbance was measured at $550 \mathrm{~nm}$ in an automated plate reader. Concentration was determined with reference to a standard curve of $\mathrm{NaNO}_{2}$.

\section{Isolation of mitochondria}

Cells were harvested with PBS using cell scraper. The pellet was resuspended in $3 \mathrm{~mL}$ of ice-cold $\mathrm{IB}_{\mathrm{c}}$ buffer (10 mM Tris, $1 \mathrm{mM}$ EGTA, $200 \mathrm{mM}$ sucrose, $10 \mathrm{mM}$ MOPS, $\mathrm{pH}$ 7.4) after washing one time with PBS. The cells were homogenized with $30-40$ strokes using a Teflon pestle. The homogenate was then transferred to a $15 \mathrm{~mL}$ polypropylene Falcon tube and centrifuged at $600 \mathrm{~g}$ for $10 \mathrm{~min}$ at $4^{\circ} \mathrm{C}$. The supernatant was collected and transferred to a glass centrifuge tube followed by centrifugation at $7,000 \mathrm{~g}$ for $10 \mathrm{~min}$ at $4^{\circ} \mathrm{C}$. The supernatant was then discarded, and the pellet was resuspended in $500 \mu \mathrm{L}$ of ice-cold $\mathrm{IB}_{\mathrm{c}}$ buffer and transferred the suspension to a $1.5 \mathrm{~mL}$ microfuge tube, followed by centrifugation at $7,000 \mathrm{~g}$ for $10 \mathrm{~min}$ at $4^{\circ} \mathrm{C}$. The pellet containing mitochondria was collected, resuspended in minimal volume of $\mathrm{IB}_{\mathrm{c}}$ buffer, and kept on ice.

\section{Mixed lymphocyte reaction (MLR)}

Control vector (pDMyc) and pDC-CASPIC-Myc transfected DC2.4 cells were harvested, counted and seeded for $4 \mathrm{~h}$ before induction (or without induction as control) with E.coli LPS (Invitrogen) $(100 \mathrm{ng} / \mathrm{mL}$ to $500 \mathrm{ng} / \mathrm{mL}$ ) for $18-24 \mathrm{~h}$. DC2.4 cells were harvested using $0.5 \mathrm{mM}$ EDTA and counted. Three million cells were fixed on ice for $30 \mathrm{~min}$ using $4 \%$ paraformaldehyde. After fixation, the DC2.4 cells were counted and mixed with thymic lymphocytes in 24-well plate at a DC to $T$ lymphocytes ratio of $1: 20\left(2.5 \times 10^{5} \mathrm{DC}\right.$ to $5 \times 10^{6}$ thymic lymphocytes). To prepare thymic lymphocytes, thymuses were extracted from Balb/C mice (7-8 weeks old) and thymic lymphocytes were isolated after subjecting the thymic cell suspension to ficollpaque plus (Amersham Biosciences) centrifugation. Thymic lymphocytes were counted, mixed with the fixed DC2.4 cells in 24-well plate, and cultured for $3 \mathrm{~d}$ before the culture supernatant were collected and used for IL-2 ELISA (Biolegend) according to the manufacturer's instructions.

\section{Statistics}

Student's $t$-test was used for statistical analyses.

\section{ACKNOWLEDGMENTS}

We thank Dr. Kenneth Rock for the DC2.4 cell line and Prof. Jack Strominger, Prof. Michael Kemeny, Dr. Lim Kah Leong and members of the Membrane Trafficking and Immunoregulation Research Laboratory for critical reading of the manuscript. This work was supported by the Academic Research Council-Singapore Ministry of Education (R182-000-099-112 to S.H.W) and NMRC (R182-000-137213 to S.H.W) grants.

\section{ABBREVIATIONS}

CARD, caspase recruitment domain; $\mathrm{Cl}$, caspase-inhibitor; CIITA, MHC class II transactivator; COX, cytochrome oxidase; DC, dendritic cells; DC-CASPIC, DC-expressed caspase inhibitory isoform of CIITA; LPS, lipopolysaccharides; MHC, major histocompatibility complex; MLR, mixed lymphocyte reaction; NO, nitric oxide; NOS2, nitric oxide synthase; ORF, open reading frame; pDCs, plasmacytoid DCs; RT-PCR, reverse-transcriptase-PCR; TEC, thymic epithelial cells

\section{REFERENCES}

Arques, J.L., Hautefort, I., Ivory, K., Bertelli, E., Reqoli, M., Clare, S., Hinton, J.C., and Nocoletti. C. (2009). Salmonella induces flagellinand MyD88-dependent migration of bacteria-capturing dendritic cells into the gut lumen. Gastroenterology 137, 579-587.

Banchereau, J., Briere, F., Caux, C., Davoust, J., Lebecque, S., Liu, Y. J., Pulendran, B., and Palucka, K. (2000). Immunobiology of 
dendritic cells. Annu Rev Immunol 18, 767-811.

Boss, J.M., and Jensen, P.E. (2003). Transcriptional regulation of the $\mathrm{MHC}$ class II antigen presentation pathway. Curr Opin Immunol 15, 105-111.

Boveris, A., Costa, L., Cadenas, E., and Poderoso, J.J. (1999). Regulation of mitochondrial respiration by adenosine phosphate, oxygen, and nitric oxide. Methods Enzymol 301, 188-198.

Boveris, A., D'Amico, G., Lores-Arnaiz, S., and Costa, L.E. (2003). Enalapril increases mitochondrial nitric oxide synthase activity in heart and liver. Antioxid Redox Signal 5, 691-697.

Brown, G.C. (1995). Nitric oxide regulates mitochondrial respiration and cell functions by inhibiting cytochrome oxidase. FEBS Lett 369, 136-139.

Brüne, B. (2003). Nitric oxide: NO apoptosis or turning it on? Cell Death Differ 10, 864-869.

Bustamante, J., Bersier, G., Romero, M., Badin, R.A., and Boveris, A. (2000). Nitric oxide production and mitochondrial dysfunction during rat thymocyte apoptosis. Arch Biochem Biophys 376, 239-247.

Carreras, M.C., Peralta, J.G., Converso, D.P., Finocchietto, P.V., Rebagliati, I., Zaninovich, A.A., and Poderoso, J.J. (2001). Modulation of liver mitochondrial NOS is implicated in thyroiddependent regulation of $\mathrm{O}(2)$ uptake. Am J Physiol Heart Circ Physiol 281, H2282-2288.

Carreras, M.C., Converso, D.P., Lorenti, A.S., Barbich, M., Levisman, D.M., Jaitovich, A., Antico Arciuch, V.G., Galli, S., and Poderoso, J. J. (2004). Mitochondrial nitric oxide synthase drives redox signals for proliferation and quiescence in rat liver development. Hepatology 40, 157-166.

Cressman, D.E., O'Connor, W.J., Greer, S.F., Zhu, X.S., and Ting, J.P. (2001). Machanisms of nuclear import and export that control the subcellular localization of class II transactivator. J Immunol 167, 3626-3634.

Dawn, B., and Bolli, R. (2002). Role of nitric oxide in myocardial preconditioning. Ann Ny Acad Sci 962, 18 41.

Franco, M.C., Antico Arciuch, V.G., Peralta, J.G., Galli, S., Levisman, D., Lopez, L.M., Ronorini, L., Poderoso, J.J., and Careras, M.C. (2006). Hypothyroid phenotype is contributed by mitochondrial complex I inactivation due to translocated neural nitric oxide synthase. J Biol Chem 281, 4779-4786.

Ghafourifar, P., and Richter, C. (1997). Nitric oxide synthase activity in mitochondria. FEBS Lett 418, 291-296.

Giulivi, C., Poderoso, J.J., and Boveris, A. (1998). Production of nitric oxide by mitochondria. J Biol Chem 273, 11038-11043.

Hiller, M., and Platzer, M. (2008). Widespread and subtle: alternative splicing at short-distance tandem sites. Trends Genet 24, 246-255.

Hong G.S. and Jung, Y.K. (2002). Caspase recruitment domain (CARD) as a bi-functional switch of caspase regulation and NF-kB signals. J Biochem Mol Biol 35, 19-23.

Huang, D., Cai, D.T., Chua, R.Y.R., Kemeny, D.M., and Wong, S.H. (2008). Nitric-oxide synthase 2 interacts with CD74 and inhibits its cleavage by caspase during dendritic cell development. J Biol Chem 283, 1713-1722.

Kim, P.K., Kwon, Y.G., Chung, H.T., and Kim, Y.M. (2002). Regulation of caspases by nitric oxide. Am NY Acad Sci 962, 42-52.

Kozak, M. (2001). Constraints on reinitiation of translation in mammals. Nucleic Acid Res 29, 5226-5232.

Kozak, M. (2002). Pushing the limits of the scanning mechanism for initiation of translation. Gene 299, 1-34.

Landmann, S., Muhlethaler-Mottet, A., Bernascori, L., Suter, T., Waldburger, J.M., Masternak, K., Arriqhi, J.F., Hauser, C., Fontana, A., and Reith, W. (2001). Maturation of dendritic cells is accompanied by rapid transcriptional silencing of class II transactivator (CIITA) expression. J Exp Med 194, 379-391.

Leibundgut-Landmann,S., Waldburger, J.M., Krawczyk, M., Otten, L. A., Suter, T., Fontana, A., Cha-Orbea, H., and Reith, W. (2004). Mini-review: Specificity and expression of CIITA, the master regulator of MHC class II genes. Eur J Immunol 34, 1513-1525.

Mori Y., Matsubara, H., Murasawa, S., Kijima, K., Maruyama, K., Tsukaguchi, H., Okubo, N., Hamakubo, T., Inagami, T., Iwasaka, T., et al. (1996). Translation regulation of angiotensin II type1A receptor. Role of upstream AUG triplets. Hypertension 28, 810-817.

Muhlethaler-Mottet,A., Otten, L.A., Steimle, V., and Mach, M. (1997). Expression of $\mathrm{MHC}$ class II molecules in different cellular and functional compartments is controlled by differential usage of multiple promoters of the transactivator CIITA. EMBO J 16, 2851-2860.

Nathan, C. (1992) Nitric oxide as a secretory product of mammalian cells. FASEB J 6, 3051-3064.

Newman, M.Y., and William, M.F.L. (1997). Coding elements in exon 2 and 3 target c-myc mRNA downregulation during myogenic differentiation. Mol Cell Biol 17, 2698-2707.

Newman, M.Y., Muhammad, T.R., and William, M.F.L. (1996). Identification of sequences in c-myc mRNA that regulate its steady-state levels. Mol Cell Biol 16, 3511-3522.

Nickerson, K., Sisk, T.J., Inohara, N., Yee, C.S., Kennell, J., Cho, M. C., Yannie, P.J., Nunez, G., and Chang, C.H. (2001). Dendritic cellspecific MHC class II transactivator contains a caspase recruitment domain that confers potent transactivation activity. J Biol Chem 276, 19089-19093.

Persichini, T., Mazzaone, V., Polticelli, F., Moreno, S., Venturini, G., Clementi, E., and Colasanti, M. (2005). Mitochondrial type I nitric oxide synthase physically interacts with cytochrome c oxidase. Neurosci Lett 384, 254-259.

Reith, W. and Mach, B. (2001). The bare lymphocyte syndrome and the regulation of MHC expression. Ann Rev Immunol 19, 331-373.

Riobo, N., Melari, M., Sanjuan, N., Carreras, M.C., Cadenas, E., and Poderoso, J.J. (2002). The modulation of mitochondrial nitric oxide synthase activity in rat brain development. J Biol Chem 277, 42447-42455.

Santambrogio, L., Potolicchio, I., Fessler, S.P., Wong, S.H., Raposo, G., and Strominger, J.L. (2005). Involvement of caspase-cleaved and intact adaptor protein 1 complex in endosomal remodeling in maturing dendritic cells. Nat Immunol 6, 1020-1028.

Ting, J.P.-Y. and Trowsdale, J. (2002). Genetic control of MHC Class II expression. Cell 109, S21-33.

Wong, S.H., Santambrogio, L., and Strominger, J.L. (2004). Caspases and nitric oxide broadly regulate dendritic cell maturation and surface expression of class II MHC proteins. Proc Natl Acad Sci U S A 101, 17783-17788.

Xu, G., Rabadan-Diehl, C., Nikodemova, M., Wynn, P., Spiess, J., and Aquilera, G. (2001). Inhibition of corticotrophin releasing hormone type I receptor translation by an upstream AUG triplet in the 5' untranslated region. Mol Pharmacol 59, 485-492.

Yeilding N.M., and Lee W.M. (1997). Coding elements in exons 2 and 
3 target c-myc mRNA downregulation during myogenic differentiation. Mol Cell Biol 17, 2698-2707.

Yeilding N.M., Rehman M.T., and Lee W.M. (1996). Identification of sequences in c-myc mRNA that regulate its steady-state levels. Mol Cell Biol 16, 3511-3522.
Zhao, X., Chen, Y.R., He, G., Zhang, A., Druhan, L.J., Strauch, A.R., and Zweier, J.L. (2007). Endothelial nitric oxide synthase (NOS3) knockout decreases NOS2 induction, limiting hyperoxygenation and confering protection in the postischemic heart. Am J Physiol Heart Circ Physiol 292, H1541-1550. 